\title{
Virtual Element Method for the Laplace-Beltrami equation on surfaces
}

\author{
Massimo Frittelli ${ }^{1}$ and Ivonne Sgura ${ }^{1}$ \\ ${ }^{1}$ Dipartimento di Matematica e Fisica E. De Giorgi, Universit del Salento, via per Arnesano, I-73100 Lecce, Italy
}

\begin{abstract}
We present and analyze a Virtual Element Method (VEM) of arbitrary polynomial order $k \in \mathbb{N}$ for the Laplace-Beltrami equation on a surface in $\mathbb{R}^{3}$. The method combines the Surface Finite Element Method (SFEM) [Dziuk, Elliott, Finite element methods for surface PDEs, 2013] and the recent VEM [Beirao da Veiga et al, Basic principles of Virtual Element Methods, 2013] in order to handle arbitrary polygonal and/or nonconforming meshes. We account for the error arising from the geometry approximation and extend to surfaces the error estimates for the interpolation and projection in the virtual element function space. In the case $k=1$ of linear Virtual Elements, we prove an optimal $H^{1}$ error estimate for the numerical method. The presented method has the capability of handling the typically nonconforming meshes that arise when two ore more meshes are pasted along a straight line. Numerical experiments are provided to confirm the convergence result and to show an application of mesh pasting.
\end{abstract}

\section{MSC Subject Classification}

$65 \mathrm{~N} 15,65 \mathrm{~N} 30$

\section{Keywords}

Surface PDEs, Laplace-Beltrami equation, Surface Finite Element Method, Virtual Element Method

\section{Introduction}

The Virtual Element Method (VEM) is a recent extension of the well-known Finite Element Method (FEM) for the numerical approximation of several classes of partial differential equations on planar domains [1-7]. The main features of the method have been introduced in $[1,8]$.

The key feature of VEM is that of being a polygonal finite element method, i.e. the method handles elements of quite general polygonal shape, rather than just triangular [1], and nonconforming meshes $[1,9]$. The increased mesh generality provides different advantages, we mention some of them. Nonconforming meshes (i) naturally arise when pasting several meshes to obtain a polygonal approximation of the whole domain $[10,11]$, as there is no need to match the nodal points in contrast to conforming pasting techniques [12,13] and (ii) allow simple adaptive refinement strategies [14]. Elements of more general shape and arbitrary number of edges allow (i) flexible approximation of the domain and in particular of its boundary [15] and (ii) the possibility of enforcing higher regularity to the numerical solution $[6,16,17]$.

The core idea of the VEM is that, given a polynomial order $k \in \mathbb{N}$ and a polygonal element $K$, the local basis function space on $K$ includes the polynomials of degree $k$ (thus ensuring the optimal degree of accuracy) plus other basis functions that are not known in closed form [1]. The presence of these virtual functions motivates the name of the method. However, the knowledge of certain degrees 
of freedom attached to the basis functions is sufficient to compute the discrete bilinear forms with a degree of accuracy $k$.

The VEM, introduced for the Laplace equation in two dimensions in the recent publication [1], has been extended to more complicated PDEs, for example a non exhaustive list is: linear elasticity [2], plate bending [17], fracture problems [7], eigenvalue problems [3], Cahn-Hilliard equation [6], heat [4] and wave equations [5].

The aim of the present work is to extend the VEM to solve surface PDEs, i.e. PDEs having a twodimensional smooth surface in $\mathbb{R}^{3}$ as spatial domain. Surface PDEs arise in the modelling of several problems such as advection [18], water waves [19], phase separation [20], reaction-diffusion systems and pattern formation [21-25], tumor growth [26], biomembrane modelling [27], cell motility [28], superconductivity [29], metal dealloying [30], image processing [21] and surface modelling [31]. We will focus on the Laplace-Beltrami equation, that is the prototypal second order elliptic PDE on smooth surfaces and corresponds to the extension of the Laplace equation to surfaces [32, chapter 14].

Among the various discretisation techniques for surface PDEs existing in literature (see for example $[24,26,33-35])$ we consider the Surface Finite Element Method (SFEM) introduced in the seminal paper [36]. The core idea is to approximate the surface with a polygonal surface made, as in the planar case, of triangular non-overlapping elements whose vertices belong to the surface and to consider a space of piecewise linear functions. The resulting method is exactly similar to the well-known planar FEM, but the convergence estimates must account for the additional error arising from the approximation of the surface, see [34] for a thorough analysis of the method. In this paper, we define a Virtual Element Method on polygonal surfaces by combining the approaches of VEM and SFEM, the resulting method will be defined as Surface Virtual Element Method (SVEM). Then we prove, under minimal regularity assumptions on the polygonal mesh, some error estimates for the for the approximation of surfaces and for the projection operators and bilinear forms involved in the method. Furthermore, we prove existence and uniqueness of the discrete solution and a first order (and thus optimal) $H^{1}$ error estimate. As an application, we show that the method simply handles composite meshes arising from pasting two (or more) meshes along a straight line.

The structure of the paper is as follows. In Section 1 we recall some preliminaries on differential operators and function spaces on surfaces. In Section 2 we recall the Laplace-Beltrami equation on arbitrary smooth surfaces without boundary in strong and weak forms. In Section 3 we introduce a Virtual Element Method for the Laplace-Beltrami equation, defined on general polygonal approximation of surfaces and for any polynomial order $k \in \mathbb{N}$. In Section 4 we prove error estimates for the discrete bilinear forms and the approximation of geometry. In Section 5 we prove existence, uniqueness and first order $H^{1}$ convergence of the numerical solution. In Section 6 we discuss the application of the method to mesh pasting. In Section 7 we face with the issues related to the implementation of the method. In Section 8 we present two numerical examples to (i) test the order of convergence of the method and (ii) show the application of the method to mesh pasting.

\section{Differential operators on surfaces}

In this section we recall some fundamental notions concerning surface PDEs. If not explicitly stated, definitions and results are taken from [34].

Definition 1 ( $\mathcal{C}^{k}$ surface, normal and conormal vectors). Given $k \in \mathbb{N}$, a set $\Gamma \subset \mathbb{R}^{3}$ is said to be $a \mathcal{C}^{k}$ surface if, for every $\mathbf{x}_{0} \in \Gamma$, there exist an open set $U_{\mathbf{x}_{0}} \subset \mathbb{R}^{3}$ containing $\mathbf{x}_{0}$ and a function $\phi_{\mathbf{x}_{0}} \in \mathcal{C}^{k}(U)$ such that

The vector field

$$
U_{\mathbf{x}_{0}} \cap \Gamma=\left\{\mathbf{x} \in U_{\mathbf{x}_{0}} \mid \phi_{\mathbf{x}_{0}}(\mathbf{x})=0\right\} .
$$

$$
\nu: \Gamma \rightarrow \mathbb{R}^{3}, \mathbf{x} \mapsto \frac{\nabla \phi_{\mathbf{x}}(\mathbf{x})}{\left\|\nabla \phi_{\mathbf{x}}(\mathbf{x})\right\|}
$$

is said to be the unit normal vector. We denote by $\partial \Gamma$ the one-dimensional boundary of $\Gamma$. If $\partial \Gamma$ has a well-defined tangent direction at each point, the vector field $\mu: \partial \Gamma \rightarrow \mathbb{R}^{3}$ such that 
- $\mu(\mathbf{x}) \perp \nu(\mathbf{x}) \quad \forall \mathbf{x} \in \partial \Gamma$;

- $\mu(\mathbf{x}) \perp \partial \Gamma \quad \forall \mathbf{x} \in \partial \Gamma$

- $\mu(\mathbf{x})$ points outward of $\Gamma$,

is called the conormal unit vector.

Lemma 1 (Fermi coordinates). If $\Gamma$ is a $\mathcal{C}^{2}$ surface, there exists an open set $U \subset \mathbb{R}^{3}$ such that every $\mathbf{x} \in U$ admits a unique decomposition of the form

$$
\mathbf{x}=\mathbf{a}(\mathbf{x})+d(\mathbf{x}) \nu(\mathbf{a}(\mathbf{x})), \quad \mathbf{a}(\mathbf{x}) \in \Gamma, d(\mathbf{x}) \in \mathbb{R} .
$$

The set $U$ is called the Fermi stripe of $\Gamma$ and $(\mathbf{a}(\mathbf{x}), d(\mathbf{x}))$ are called the Fermi coordinates of $\mathbf{x}$.

Definition 2 (Tangential gradient, tangential divergence). If $\Gamma$ is a $\mathcal{C}^{1}$ surface, $A$ is an open neighborhood of $\Gamma$ and $f \in \mathcal{C}^{1}(A, \mathbb{R})$, the operator

$$
\nabla_{\Gamma} f: S \rightarrow \mathbb{R}^{3}, \mathbf{x} \mapsto \nabla f(\mathbf{x})-(\nabla f(\mathbf{x}) \cdot \nu(\mathbf{x})) \nu(\mathbf{x})=P(\mathbf{x}) \nabla f(\mathbf{x}),
$$

where $P(\mathbf{x})_{i j}=\delta_{i j}-\nu_{i}(\mathbf{x}) \nu_{j}(\mathbf{x})$, is called the tangential gradient of $f$. The components of the tangential gradient, i.e.

$$
\underline{D}_{i} f: S \rightarrow \mathbb{R}, \mathbf{x} \mapsto P_{i}(\mathbf{x}) \nabla f(\mathbf{x}), \quad i \in\{1,2,3\},
$$

where $P_{i}(\mathbf{x})$ is the $i$-th row of $P(\mathbf{x})$, are called the tangential derivatives of $f$. Given a vector field $F \in \mathcal{C}^{1}\left(A, \mathbb{R}^{3}\right)$, the operator

$$
\nabla_{\Gamma} \cdot F: S \rightarrow \mathbb{R}, \mathbf{x} \mapsto \sum_{i=1}^{3} \underline{D}_{i} F_{i}(\mathbf{x})
$$

is called the tangential divergence of $F$.

Theorem 1. Given $\Gamma \subset A$ a $\mathcal{C}^{1}$ surface, if $f$ and $g$ are $\mathcal{C}^{1}(A, \mathbb{R})$ functions such that $f_{\mid \Gamma}=g_{\mid \Gamma}$, then

$$
\nabla_{\Gamma} f(\mathbf{x})=\nabla_{\Gamma} g(\mathbf{x}) \quad \forall \mathbf{x} \in \Gamma .
$$

This means that the tangential gradient of a function only depends on its restriction over $\Gamma$.

Theorem 1 makes the following definition well-posed.

Definition $3\left(\mathcal{C}^{k}(\Gamma)\right.$ functions). If $\Gamma$ is a $\mathcal{C}^{1}$ surface, a function $f: \Gamma \rightarrow \mathbb{R}$ is said to be $\mathcal{C}^{1}(\Gamma)$ if it is differentiable at any point of $\Gamma$ and its tangential derivatives are continuous over $\Gamma$.

If $k \geq 2$ and $\Gamma$ is a $\mathcal{C}^{k}$ surface, a function $f: \Gamma \rightarrow \mathbb{R}$ is said to be $\mathcal{C}^{k}(\Gamma)$ if it is $\mathcal{C}^{1}(\Gamma)$ and its tangential derivatives are $\mathcal{C}^{k-1}(\Gamma)$ functions.

Definition 4 (Laplace-Beltrami operator). Given a $\mathcal{C}^{2}$ surface $\Gamma$ and $f \in \mathcal{C}^{2}(\Gamma)$, the operator

$$
\Delta_{\Gamma} f: \Gamma \rightarrow \mathbb{R}, \mathbf{x} \mapsto \nabla_{\Gamma} \cdot \nabla_{\Gamma} f(\mathbf{x})=\sum_{i=1}^{3} \underline{D}_{i} \underline{D}_{i} f(\mathbf{x})
$$

is called the Laplace-Beltrami operator of $f$.

We now recall the definitions of some remarkable Sobolev spaces on surfaces.

Definition 5 (Sobolev spaces on surfaces). Given $s \in \mathbb{N}$, let $\Gamma$ be a $C^{s}$ surface and let $L^{0}(\Gamma)$ be the set of measurable functions with respect to the bidimensional Hausdorff measure on $\Gamma$. Consider the Sobolev spaces

$$
\begin{aligned}
& H^{0}(\Gamma)=L^{2}(\Gamma)=\left\{f \in L^{0}(\Gamma) \mid \int_{\Gamma} f^{2} \mathrm{~d} \sigma<+\infty\right\} ; \\
& H^{r}(\Gamma)=\left\{f \in L^{2}(\Gamma) \mid \underline{D}_{i} f \in H^{r-1}(\Gamma) \forall i \in\{1,2,3\}\right\}, \quad \forall 1 \leq r \leq s ; \\
& H_{0}^{r}(\Gamma)=\left\{f \in H^{r}(\Gamma) \mid \int_{\Gamma} f=0\right\}, \quad \forall 1 \leq r \leq s,
\end{aligned}
$$


where derivatives are meant in distributional sense ${ }^{1}$. These are Hilbert spaces if endowed with the scalar products

$$
<f, g>_{H^{r}(\Gamma)}=\int_{\Gamma}\left(\sum_{|\boldsymbol{\alpha}| \leq r} \underline{D}_{\alpha} f \underline{D}_{\alpha} g\right) \mathrm{d} \sigma \quad \forall f, g \in H^{r}(\Gamma) \quad \forall 0 \leq r \leq s .
$$

where $\underline{D}_{\alpha}$ is the multi-index notation for partial tangential derivatives.

Norms will be denoted by $\|\cdot\|_{L^{2}(\Gamma)},\|\cdot\|_{H^{r}(\Gamma)}$ and seminorms by $|\cdot|_{H^{r}(\Gamma)}$.

As well as in the planar case, a Poincaré inequality holds on $H_{0}^{1}(\Gamma)$.

Theorem 2 (Poincaré's inequality on surfaces). Given a $\mathcal{C}^{2}$ surface $\Gamma$ with a well-define tangent vector field on the boundary $\partial \Gamma$, there exists $C>0$ such that

$$
\|f\|_{L^{2}(\Gamma)} \leq C|f|_{H^{1}(\Gamma)} \quad \forall f \in H_{0}^{1}(\Gamma) .
$$

A basic result in surface calculus is the following

Theorem 3 (Green's formula on surfaces). Given a $\mathcal{C}^{2}$ surface $\Gamma$ with a well-defined tangent vector field on the boundary $\partial \Gamma$ and $f, g \in \mathcal{C}^{2}(\Gamma)$, it holds

$$
\int_{\Gamma} f \Delta_{\Gamma} g \mathrm{~d} \sigma=-\int_{\Gamma} \nabla_{\Gamma} f \cdot \nabla_{\Gamma} g \mathrm{~d} \sigma+\int_{\partial \Gamma} f \frac{\partial g}{\partial \mu} \mathrm{d} l
$$

where $\frac{\partial g}{\partial \mu}(\mathbf{x})=\nabla_{\Gamma} g(\mathbf{x}) \cdot \mu(\mathbf{x})$ is the conormal derivative of $g$ on $\partial \Gamma$.

\section{The Laplace-Beltrami equation}

In this section we introduce the Laplace-Beltrami equation on a surface without boundary, that will be the model problem throughout the paper.

Let $\Gamma$ be a $\mathcal{C}^{3}$ surface without boundary and let $f \in L^{2}(\Gamma)$ such that $\int_{\Gamma} f=0$. Consider the LaplaceBeltrami equation on $\Gamma$, given by

$$
\left\{\begin{array}{c}
-\Delta_{\Gamma} u(\mathbf{x})=f(\mathbf{x}), \quad \mathbf{x} \in \Gamma \\
\int_{\Gamma} u(\mathbf{x}) \mathrm{d} \mathbf{x}=0
\end{array}\right.
$$

and its weak formulation

$$
\left\{\begin{array}{l}
u \in H_{0}^{1}(\Gamma) \\
\int_{\Gamma} \nabla_{\Gamma} u \cdot \nabla_{\Gamma} \phi=\int_{\Gamma} f \phi \quad \forall \phi \in H^{1}(\Gamma) .
\end{array}\right.
$$

Notice that, from condition $\int_{\Gamma} f=0$, the formulation (5) is equivalent to

$$
\left\{\begin{array}{l}
u \in H_{0}^{1}(\Gamma) \\
\int_{\Gamma} \nabla_{\Gamma} u \cdot \nabla_{\Gamma} \phi=\int_{\Gamma} f \phi \quad \forall \phi \in H_{0}^{1}(\Gamma) .
\end{array}\right.
$$

By considering the bilinear form $a(u, v):=\int_{\Gamma} \nabla_{\Gamma} u \cdot \nabla_{\Gamma} v$ for all $u, v \in H^{1}(\Gamma)$ and $\langle u, v\rangle_{L^{2}(\Gamma)}:=\int_{\Gamma} u v$ for all $u, v \in L^{2}(\Gamma)$, (6) becomes

$$
\left\{\begin{array}{l}
u \in H_{0}^{1}(\Gamma) \\
a(u, \phi)=\langle f, \phi\rangle_{L^{2}(\Gamma)} \quad \forall \phi \in H_{0}^{1}(\Gamma) .
\end{array}\right.
$$

Let us justify the above requirements $\int_{\Gamma} u=0$ and $\int_{\Gamma} f=0$. Since $\phi \equiv 1$ is allowed as a test function for the weak Laplace-Beltrami equation (5), it follows $\int_{\Gamma} f=0$ as a compatibility condition. Moreover, if $u$ fulfills $a(u, \phi)=\langle f, \phi\rangle_{L^{2}(\Gamma)}, \forall \phi \in H^{1}(\Gamma)$ and $c \in \mathbb{R}$, then $u+c$ fulfills the same equation; condition $\int_{\Gamma} u=0$ is thus enforced to provide uniqueness of the solution. Existence and uniqueness for problem (7) will be proven rigorously in Theorem 9 in Section 5 .

\footnotetext{
${ }^{1}$ See [37, Chapter 4] or [34, Definition 2.11] for a precise definition of distributional tangential derivatives.
} 
Remark 1 (Surfaces with boundary). The whole analysis carried out in this paper holds unchanged in the presence of a non-empty boundary, $\partial \Gamma \neq \emptyset$, and homogeneous Neumann boundary conditions. In the case of homogeneous Dirichlet boundary conditions, the analysis still holds if $H_{0}^{1}(\Gamma)$ is the space of $H^{1}(\Gamma)$ functions that vanish on $\partial \Gamma$ in a weak sense, see [37, Chapter 4.5].

\section{Space discretisation by SVEM}

In this section, we will address space discretisation of (7). After defining the approximation of geometry and the corresponding discrete function spaces, the Surface Virtual Element Method (SVEM) will be introduced.

\subsection{Approximation of the surface}

In this section we define a polygonal approximation of the surface $\Gamma$ in Definition 1 and a virtual element space on this polygonal approximation. The method will thus generalise, in the piecewise linear case, the Surface Finite Element Method (SFEM) [34] and the Virtual Element Method (VEM) [1] at once. Given a $\mathcal{C}^{2}$ surface $\Gamma$ in $\mathbb{R}^{3}$, we constuct a piecewise flat approximate surface $\Gamma_{h}$, defined as

$$
\Gamma_{h}=\bigcup_{E \in \mathcal{T}_{h}} E
$$

where

1. $\mathcal{T}_{h}$ is a finite set of non-overlapping simple polygons, i.e. without holes and with non selfintersecting boundary, having diameters less than or equal to $h>0$;

2. $\Gamma_{h}$ is contained in the Fermi stripe $U$ associated to $\Gamma$, see Lemma 1;

3. $\mathbf{a}: \Gamma_{h} \rightarrow \Gamma$ is one-to-one;

4. the vertices of $\Gamma_{h}$ lie on $\Gamma$.

Following [34], we define how to lift functions from the approximate surface $\Gamma_{h}$ to the continuous one $\Gamma$.

Definition 6 (Lifted functions). Let $\Gamma$ be a $\mathcal{C}^{2}$ surface and $\Gamma_{h}$ be as in (8). Given a function $\phi: \Gamma_{h} \rightarrow$ $\mathbb{R}$, its lift $\phi^{\ell}: \Gamma \rightarrow \mathbb{R}$ is defined by $\phi \circ\left(\mathbf{a}_{\mid \Gamma_{h}}\right)^{-1}$. Given a function $\psi: \Gamma \rightarrow \mathbb{R}$, its unlift $\psi^{-\ell}: \Gamma_{h} \rightarrow \mathbb{R}$ is defined by $\psi \circ \mathbf{a}$.

This definition is well-posed thanks to assumption (3).

Furthermore, the following mesh regularity requirements will be assumed throughout the paper. There exist $\gamma_{1}, \gamma_{2}>0$ such that, for all $h>0$ and $E \in \mathcal{T}_{h}$,

(A) $E$ is star-shaped with respect to a ball of radius $\rho_{E}$ such that

$$
\rho_{E} \geq \gamma_{1} h_{E}
$$

(B) for every pair of nodes $P, Q \in E$, the distance $\|P-Q\|$ fulfills

$$
\|P-Q\| \geq \gamma_{2} h_{E}
$$

where $h_{E}$ is the diameter of $E$.

Remark 2. This kind of polygonal approximation has two remarkable subcases:

1. If each $E \in \mathcal{T}_{h}$ has three vertices, we obtain the classical triangulations adopted, for instance, in [34] and [36].

2. If $\Gamma$ is a flat surface, we obtain the polygonal meshes considered in [1].

We remark that the considered class of polygonations includes nonconforming meshes. We will show in Section 6 that this feature can be exploited in mesh pasting. 


\subsection{Discrete function spaces}

Consider a polynomial degree $k \in \mathbb{N}$ and $E \in \mathcal{T}_{h}$. Without loss of generality, $E$ may be assumed to lie in the $(x, y)$ plane. Following [1], the local virtual space of degree $k$ in $E$ is defined by

$$
V_{h}^{k}(E)=\left\{v_{h} \in H^{1}(E) \mid v_{h \mid e} \in \mathbb{P}_{k}(e) \forall e \in \operatorname{edges}(E), \Delta v_{h} \in \mathbb{P}_{k-2}(E)\right\} .
$$

The set of barycentric polynomials on $E$

$$
\mathcal{M}_{k-2}(E)=\left\{\left(\frac{\mathbf{x}-\mathbf{x}_{E}}{h_{E}}\right)^{\boldsymbol{\alpha}}|| \boldsymbol{\alpha} \mid \leq k-2\right\},
$$

where $\mathbf{x}_{E}$ and $h_{E}$ are the barycenter and the diameter of $E$, respectively, is a basis for $\mathbb{P}_{k-2}(E)$. For every $v_{h} \in V_{h}^{k}(E)$, the following degrees of freedom are defined:

1. the pointwise value of $v_{h}$ on the $n_{E}$ vertices of $E$;

2. the pointwise value of $v_{h}$ on $k-1$ equally spaced points (different from the vertices) of each edge of $E$;

3. the $\frac{k(k-1)}{2}$ moments

$$
\frac{1}{|E|} \int_{E} v_{h} m_{k-2} \quad \forall m_{k-2} \in \mathcal{M}_{k-2}(E) .
$$

In [1] it has been proven that these degrees of freedom are unisolvent for the space $V_{h}^{k}(E)$ in $(9)$. Given $w \in H^{s}(E), s>1$, by Sobolev's embedding theorem we have $w \in \mathcal{C}^{0}(E)$. Hence, the degrees of freedom of $w$ are well-defined. If $n_{E}^{d o f}$ is the number of degrees of freedom on $E$ and $d o f_{i}\left(v_{h}\right)$ denotes the $i^{t h}$ degree of freedom of $v_{h}, i=1, \ldots, n_{E}^{d o f}$, the unique function $w_{I} \in V_{h}^{k}(E)$ such that

$$
\operatorname{dof}_{i}\left(w-w_{I}\right)=0 \quad \forall i=1, \ldots, n_{E}^{\text {dof }}
$$

is said to be the interpolant of $w$. If $\tilde{E}=\mathbf{a}(E)$ is the curved triangle corresponding to $E$ and $v \in H^{2}(\tilde{E})$, we have $v^{-\ell} \in H^{2}(E)$, see Theorem 3. The unique function $v_{I} \in V_{h}^{k}(E)$ such that

$$
\operatorname{dof}_{i}\left(v^{-\ell}-v_{I}\right)=0 \quad \forall i=1, \ldots, n_{E}^{d o f}
$$

is said to be the interpolant of $v$.

Remark 3. From the definition of $V_{h}(E)$ in (9) it follows that

1. $\mathbb{P}_{k}(E) \subseteq V_{h}^{k}(E)$;

2. every $v_{h} \in V_{h}^{k}(E)$ is explicitly known on the boundary $\partial E$, but not on the interior $\stackrel{\circ}{E}$;

3. if $k=1, V_{h}^{1}(E)$ is the set of harmonic functions in $E$ being piecewise linear on the boundary $\partial E$ and the set of local degrees of freedom collapses to the pointwise values on the vertices of $E$;

4. if $k=1$ and $E$ is a triangle, $V_{h}^{1}(E)=\mathbb{P}_{1}(E)$; this is the only case in which $V_{h}^{k}(E)=\mathbb{P}_{k}(E)$, i.e the VEM method reduces to FEM.

The global discrete space will be defined by

$$
V_{h}^{k}=\left\{v_{h} \in \mathcal{C}^{0}\left(\Gamma_{h}\right) \mid v_{h \mid E} \in V_{h}^{k}(E) \forall E \in \mathcal{T}_{h}\right\} .
$$

Furthermore, we define the zero-averaged virtual space $W_{k}^{k}$ by

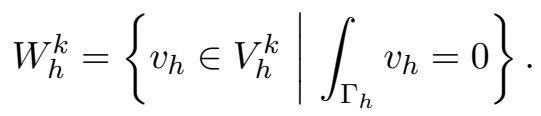

Finally, we define the following broken $H^{s}$ seminorms, $s \in\{1,2\}$, on the polygonal surface $\Gamma_{h}$ :

$$
\left|v_{h}\right|_{h, s}=\sqrt{\sum_{E \in \mathcal{T}_{h}}\left|v_{h \mid E}\right|_{H^{s}(E)}^{2}} \quad \forall v_{h} \in \prod_{E \in \mathcal{T}_{h}} H^{s}(E)
$$




\subsection{The Surface Virtual Element Method}

We may write a discrete formulation for (7):

$$
\left\{\begin{array}{l}
u_{h} \in W_{h}^{k} \\
\int_{\Gamma_{h}} \nabla_{\Gamma_{h}} u_{h} \cdot \nabla_{\Gamma_{h}} \phi_{h}=\int_{\Gamma_{h}} f_{I} \phi_{h} \quad \forall \phi_{h} \in W_{h}^{k},
\end{array}\right.
$$

where $f_{I}$ is the interpolant of $f$ defined piecewise in (11).

Remark 4 (Regularity of $f$ ). In the following we assume $f \in H^{2}(\Gamma)$, such that, from Sobolev's embedding theorem, its pointwise values (and thus its interpolant $f_{I}$ ) are well-defined. We remark that, in the framework of surface PDEs, the problem of numerically handling $H^{s}(\Gamma), 0 \leq s \leq 1$, load terms is intrinsically challenging. In fact, if the pointwise values of $f$ are not available, then any approximation $\bar{f}$ of $f$ defined on $\Gamma_{h}$ must account for the mapping $\mathbf{a}: \Gamma_{h} \rightarrow \Gamma$ in (1) that, in general, is not computable.

By introducing the bilinear forms $\bar{a}\left(u_{h}, v_{h}\right):=\int_{\Gamma_{h}} \nabla_{\Gamma_{h}} u_{h} \cdot \nabla_{\Gamma_{h}} v_{h}$ for all $u_{h}, v_{h} \in V_{h}^{k}$ and $\left\langle U_{h}, V_{h}\right\rangle_{L^{2}\left(\Gamma_{h}\right)}$ for all $U_{h}, V_{h} \in L^{2}\left(\Gamma_{h}\right)$, problem (13) is equivalent to

$$
\left\{\begin{array}{l}
u_{h} \in W_{h}^{k} \\
\bar{a}\left(u_{h}, \phi_{h}\right)=\left\langle f_{I}, \phi_{h}\right\rangle_{L^{2}\left(\Gamma_{h}\right)} \quad \forall \phi_{h} \in W_{h}^{k} .
\end{array}\right.
$$

We recall that functions in $V_{h}^{k}$ are virtual, i.e. they are not known explicitly, then $\bar{a}(\cdot, \cdot)$ and $\left\langle f_{I}, \cdot\right\rangle_{L^{2}}\left(\Gamma_{h}\right)$ are not computable. We thus need to write a computable approximation of problem (14). To this end, following [1], an approximate bilinear form $a_{h}(\cdot, \cdot)$ and an approximate linear form $\left\langle f_{h}, \cdot\right\rangle_{h}$ will be constructed instead of $\bar{a}(\cdot, \cdot)$ and $\left\langle f_{I}, \cdot\right\rangle_{L^{2}\left(\Gamma_{h}\right)}$, respectively.

Given the following decomposition of $\bar{a}$

$$
\bar{a}\left(v_{h}, w_{h}\right)=\sum_{E \in \mathcal{T}_{h}} \bar{a}_{E}\left(v_{h \mid E}, w_{h \mid E}\right) \quad \forall v_{h}, w_{h} \in V_{h}^{k},
$$

consider the projection $\Pi_{E}^{\nabla}: V_{h}^{k}(E) \rightarrow \mathbb{P}_{k}(E)$ defined by

$$
\left\{\begin{array}{l}
\Pi_{E}^{\nabla}\left(v_{h}\right) \in \mathbb{P}_{k}(E) \\
\bar{a}_{E}\left(\Pi_{E}^{\nabla}\left(v_{h}\right), q_{k}\right)=\bar{a}_{E}\left(v_{h}, q_{k}\right) \quad \forall q_{k} \in \mathbb{P}_{k}(E)
\end{array}\right.
$$

together with

$$
\left\{\begin{array}{l}
\sum_{P \in \operatorname{nodes}(E)} \Pi_{E}^{\nabla} v_{h}(P)=\sum_{P \in \operatorname{nodes}(E)} v_{h}(P) \quad \text { if } k=1, \\
\int_{E} \Pi_{E}^{\nabla} v_{h}=\int_{E} v_{h} \quad \text { if } k>1 .
\end{array}\right.
$$

The additional condition (16) is enforced to fix the free constant in (15). We now prove that $\Pi_{E}^{\nabla}$ is computable. The right hand side of (15) is computable, since

$$
\bar{a}_{E}\left(v_{h}, w_{h}\right)=\int_{E} \nabla v_{h} \cdot \nabla q_{k}=-\underbrace{\int_{E} v_{h} \Delta q_{k}}_{(\text {term 1) }}+\underbrace{\int_{\partial E} v_{h}\left(\nabla q_{k} \cdot \nu_{E}\right)}_{(\text {term 2) }},
$$

where $\nu_{E}$ is the unit outward vector on $\partial E$, and

- $\Delta q_{k}$ is a polynomial of degree $k-2$, thus (term 1) in (17) is a linear combination of the moments of $v_{h}$;

- $v_{h}$ and $q_{k}$ are both explicitly known (and polynomials) on $\partial E$. 
The right hand side of (16) is computable since

- for $k=1$, it is a combination of the pointwise values, $v_{h}(P), P \in \operatorname{nodes}(E)$, that are degrees of freedom;

- for $k>1$, it is one of the moments of $v_{h}$, as the space of barycentric monomials $\mathcal{M}_{k-2}$ contains the constant monomial $m_{k-2} \equiv 1$.

Hence, $\Pi_{E}^{\nabla}$ is computable. By expressing $v_{h}, w_{h} \in V_{h}^{k}(E)$ as

$$
\begin{aligned}
& v_{h}=\Pi_{E}^{\nabla} v_{h}+\left(I-\Pi_{E}^{\nabla}\right) v_{h} ; \\
& w_{h}=\Pi_{E}^{\nabla} w_{h}+\left(I-\Pi_{E}^{\nabla}\right) v_{w},
\end{aligned}
$$

the form $\bar{a}_{E}\left(v_{h}, w_{h}\right)$ may be decomposed as

$$
\bar{a}_{E}\left(v_{h}, w_{h}\right)=\underbrace{\bar{a}_{E}\left(\Pi_{E}^{\nabla} v_{h}, \Pi_{E}^{\nabla} w_{h}\right)}_{(\text {term 1) }}+\underbrace{\bar{a}_{E}\left(\left(I-\Pi_{E}^{\nabla}\right) v_{h},\left(I-\Pi_{E}^{\nabla}\right) w_{h}\right)}_{(\text {term 2) }} \quad \forall v_{h}, w_{h} \in V_{h}^{k}(E),
$$

because cross-terms vanish due to the definition of $\Pi_{E}^{\nabla}$. Notice that (term 1) in (20) is computable since $\Pi_{E}^{\nabla}$ is computable, but does not scale as $\bar{a}_{E}$ on $\operatorname{ker}\left(\Pi_{E}^{\nabla}\right)$. Then (term 2) in (20) cannot be neglected, but must be approximated in a suitable way. To this end we recall that, under the regularity assumptions (A)-(B), the bilinear form

$$
S_{E}\left(v_{h}, w_{h}\right)=\sum_{i=1}^{n_{E}^{d o f}} \operatorname{dof} f_{i}\left(v_{h}\right) d o f_{i}\left(w_{h}\right) \quad \forall v_{h}, w_{h} \in V_{h}^{k}(E),
$$

scales as $\bar{a}_{E}$ on the kernel of $\Pi_{E}^{\nabla}$, i.e. there exist $c^{*}>c_{*}>0$ such that

$$
c_{*} \bar{a}_{E}\left(v_{h}, v_{h}\right) \leq S_{E}\left(v_{h}, v_{h}\right) \leq c^{*} \bar{a}_{E}\left(v_{h}, v_{h}\right) \quad \forall v_{h} \in \operatorname{ker}\left(\Pi_{E}^{\nabla}\right),
$$

see [1]. Consider now a local approximate form $a_{h, E}$ defined by

$$
a_{h, E}\left(v_{h}, w_{h}\right)=\bar{a}_{E}\left(\Pi_{E}^{\nabla} v_{h}, \Pi_{E}^{\nabla} w_{h}\right)+S_{E}\left(\left(I-\Pi_{E}^{\nabla}\right) v_{h},\left(I-\Pi_{E}^{\nabla}\right) w_{h}\right) \quad \forall v_{h}, w_{h} \in V_{h}^{k}(E) .
$$

Notice that, since $\Pi_{E}^{\nabla} q_{k}=q_{k}$ for all $q_{k} \in \mathbb{P}_{k}(E)$, the local form (23) satisfes the consistency property

$$
a_{h, E}\left(v_{h}, q_{k}\right)=\bar{a}_{E}\left(v_{h}, q_{k}\right) \quad \forall v_{h} \in V_{h}^{k}(E), \quad \forall q_{k} \in \mathbb{P}_{k}(E) .
$$

A global approximate gradient form defined by pasting the local ones:

$$
a_{h}\left(v_{h}, w_{h}\right)=\sum_{E \in \mathcal{T}_{h}} a_{h, E}\left(v_{h \mid E}, w_{h \mid E}\right) \quad \forall v_{h}, w_{h} \in V_{h}^{k}
$$

We want to define an approximate $L^{2}$ form and the approximate right hand side. For $n \geq 0$ and for every $E \in \mathcal{T}_{h}$, consider the local $L^{2}(E)$ projection $\Pi_{n}^{E}: V_{h}^{k}(E) \rightarrow \mathbb{P}_{n}(E)$ given by

$$
\left\{\begin{array}{l}
\Pi_{n}^{E}\left(v_{h}\right) \in \mathbb{P}_{n}(E), \\
\left\langle\Pi_{n}^{E} v_{h}, q_{n}\right\rangle_{L^{2}(E)}=\left\langle v_{h}, q_{n}\right\rangle_{L^{2}(E)} \quad \forall q_{n} \in \mathbb{P}_{n}(E) .
\end{array}\right.
$$

In (26) we choose $n$ depending on $k$ as

$$
n_{k}=\left\{\begin{array}{lll}
0 & \text { if } & k=1 \\
k-2 & \text { if } & k \geq 2
\end{array}\right.
$$

We remark that 
- for $k=1$, we have that $\Pi_{0}^{E}=\Pi_{E}^{\nabla}$ (see for instance [8]), hence $\Pi_{0}^{E}$ is computable;

- for $k \geq 2, \Pi_{k-2}^{E}$ is computable since, in $(26),\left\langle v_{h}, q_{k-2}\right\rangle_{L^{2}(E)}$ is a linear combination of the moments of $v_{h}$.

Following [8], and in analogy with the approximate gradient form (23), we consider the following local approximate $L^{2}$ form:

$$
\left\langle v_{h}, w_{h}\right\rangle_{L_{h, E}^{2}}:=\int_{\Gamma_{h}} \Pi_{n_{k}}^{E} v_{h} \Pi_{n_{k}}^{E} w_{h}+S_{E}\left(\left(I-\Pi_{n_{k}}^{E}\right) v_{h},\left(I-\Pi_{n_{k}}^{E}\right) w_{h}\right), \quad \forall v_{h}, w_{h} \in V_{h}^{k}(E),
$$

where $S_{E}$ and $\Pi_{n_{k}}^{E}$ are defined in (21) and (26), respectively. Notice that the approximate $L^{2}$ form (28) fulfills the consistency property.

$$
\left\langle v_{h}, q_{n_{k}}\right\rangle_{L_{h, E}^{2}}=\left\langle v_{h}, q_{n_{k}}\right\rangle_{L^{2}(E)}, \quad \forall v_{h} \in V_{h}^{k}(E), \quad \forall q_{n_{k}} \in \mathbb{P}_{n_{k}}(E) .
$$

As a consequence, for any $k \in \mathbb{N}$ we have that

$$
\left\langle v_{h}, 1\right\rangle_{L_{h}^{2}, E}=\int_{E} v_{h} \quad \forall v_{h} \in V_{h}^{k}(E),
$$

i.e. the integral of any $V_{h}^{k}$ function can be computed exactly. A computable global approximate $L^{2}$ form is obtained by pasting the local ones:

$$
\left\langle v_{h}, w_{h}\right\rangle_{L_{h}^{2}}=\sum_{E \in \mathcal{T}_{h}}\left\langle v_{h \mid E}, w_{h \mid E}\right\rangle_{L_{h, E}^{2}}, \quad \forall v_{h}, w_{h} \in V_{h}^{k}
$$

Property (27) implies that the space $W_{h}^{k}$ defined in (12) can be represented as

$$
W_{h}^{k}=\left\{v_{h} \in V_{h} \mid\left\langle v_{h}, 1\right\rangle_{L_{h}^{2}}=0\right\},
$$

hence $W_{k}$ is computable. To approximate the right hand side, following [1], for any function $g \in H^{1}\left(\Gamma_{h}\right)$ we consider the functional $\langle g, \cdot\rangle_{h}$ defined by

$$
\left\langle g, v_{h}\right\rangle_{h}=\left\{\begin{array}{ll}
\sum_{E \in \mathcal{T}_{h}} \int_{E} g \sum_{V \in \operatorname{nodes}(E)} \frac{v_{h}(V)}{n_{E}} & \text { if } k=1 \\
\sum_{E \in \mathcal{T}_{h}} \int_{E} \Pi_{k-2}^{E} g v_{h} & \text { if } k \geq 2
\end{array} \quad \forall v_{h} \in V_{h}^{k},\right.
$$

where $n_{E}$ is the number of nodes of $E$. From (27) we have that, if $g \in V_{h}^{k},\left\langle g, v_{h}\right\rangle_{h}$ is computable, given the degrees of freedom of $g$ and $v_{h}$. Furthermore, notice that

$$
\langle g, 1\rangle_{h}=\int_{\Gamma_{h}} g, \quad \forall g \in H^{1}(\Gamma) .
$$

In order to simplify the implementation, as we will discuss in Section 7 , we define an approximate computable load term $f_{h}:=f_{I}-\frac{\left\langle f_{I}, 1\right\rangle_{L_{h}^{2}}}{\left|\Gamma_{h}\right|}$. From (27) it follows that $f_{h}$ is zero averaged and, from (31), $f_{h}$ fulfills

$$
\left\langle f_{h}, 1\right\rangle_{h}=0
$$

We may now write a computable discrete problem:

$$
\left\{\begin{array}{l}
u_{h} \in W_{h}^{k} \\
a_{h}\left(u_{h}, \phi_{h}\right)=\left\langle f_{h}, \phi_{h}\right\rangle_{h} \quad \forall \phi_{h} \in W_{h}^{k} .
\end{array}\right.
$$

We will discuss the implementation of (33) in Section 7.

The error analysis will be carried out in the following steps:

1. the geometric and interpolation error estimates in [34] will be extended to our polygonal/virtual setting;

2. the error between the continuous weak formulation (7) and the computable discrete one (33) will be estimated by extending the analogous convergence theorem in [1].

In Section 4 we deal with step (1), in Section 5 we deal with step (2). 


\section{Interpolation, projection and geometric error estimates}

We start this section by recalling some results from [1]. The following theorem addresses the projection error on $\mathbb{P}_{k}(E), E \in \mathcal{T}_{h}$.

Theorem 4. Under the regularity assumption (A), there exists $C>0$, depending only on $k$ and $\gamma$, such that for every $1 \leq s \leq k+1$ and for all $w \in H^{s}(E)$ there exists a $w_{\pi} \in \mathbb{P}_{k}(E)$ such that

$$
\left\|w-w_{\pi}\right\|_{L^{2}(E)}+h_{E}\left|w-w_{\pi}\right|_{H^{1}(E)} \leq C h_{E}^{s}|w|_{H^{s}(E)}
$$

We now address interpolation in $V_{k}(E), E \in \mathcal{T}_{h}$. The following theorem from [1] gives an interpolation error estimate in $V_{h}^{k}(E)$.

Theorem 5. Under the regularity assumption (A), there exists $C>0$, depending only on $k$ and $\gamma$, such that for every $2 \leq s \leq k+1$ and for all $w \in H^{s}(E)$, the interpolant $w_{I} \in V_{h}^{k}(E)$ satisfies

$$
\left\|w-w_{I}\right\|_{L^{2}(E)}+h\left|w-w_{I}\right|_{H^{1}(E)} \leq C h_{E}^{s}|w|_{H^{s}(E)} .
$$

To approximate a function $u: \Gamma \rightarrow \mathbb{R}$ with an interpolant defined on $\Gamma_{h}$ as in (11), also a geometric error has to be taken into account. The following lemma generalizes Lemma 4.1 in [34] to our polygonal approximations of the surface.

Lemma 2. Let $\Gamma_{h}$ be a polygonal approximation of $\Gamma$ as in (8). For any $E \in \mathcal{T}_{h}$, the oriented distance function introduced in (1) fulfills

$$
\|d\|_{L^{\infty}(E)} \leq C h^{2} .
$$

The quotient $\delta_{h}$ between the smooth and the discrete surface measures defined by $\mathrm{d} A=\delta_{h} \mathrm{~d} A_{h}$ satisfies

$$
\left\|1-\delta_{h}\right\|_{L^{\infty}\left(\Gamma_{h}\right)} \leq C h^{2} .
$$

Let $P$ and $P_{h}$ be the projections onto the tangent planes of the smooth and the discrete surfaces, respectively, that is $P_{i j}=\delta_{i j}-\nu_{i} \nu_{j}, P_{h, i j}=\delta_{i j}-\nu_{h, i} \nu_{h, j}$, and define

$$
R_{h}=\frac{1}{\delta_{h}} P(I-d \mathcal{H}) P_{h}(I-d \mathcal{H}),
$$

where $\mathcal{H}$ is the Weingarten map defined by $\mathcal{H}_{i j}=\frac{\mathrm{d} \nu_{i}}{\mathrm{~d} x_{j}}$. Then

$$
\left\|\left(I-R_{h}\right) P\right\|_{L^{\infty}\left(\Gamma_{h}\right)} \leq C h^{2} .
$$

In all of the claimed inequalities $C$ depends only on the curvature of $\Gamma$.

Proof. Consider $E \in \mathcal{T}_{h}$, see Fig. 1(a). First of all we prove that

$$
\|d\|_{L^{\infty}(\partial E)} \leq \frac{h^{2}}{8}|d|_{\mathcal{C}^{2}(E)} .
$$

To this end, let $\mathbf{x}_{B} \in \partial E$ and let $e$ be an edge of $E$ such that $\mathbf{x}_{B} \in e$, see Fig. 1(b). Then, if $d_{e}$ is the linear interpolant of $d$ on $e$, from the classical Lagrange interpolation estimates and from the fact that $d_{e} \equiv 0$ since $d$ vanishes at the endpoints of $e$, we have

$$
\left|d\left(\mathbf{x}_{B}\right)\right| \leq\left\|d_{e}\right\|_{L^{\infty}(e)}+\left\|d-d_{e}\right\|_{L^{\infty}(e)} \leq \frac{|e|^{2}}{8}|d|_{\mathcal{C}^{2}(e)} \leq \frac{h^{2}}{8}|d|_{\mathcal{C}^{2}(E)},
$$




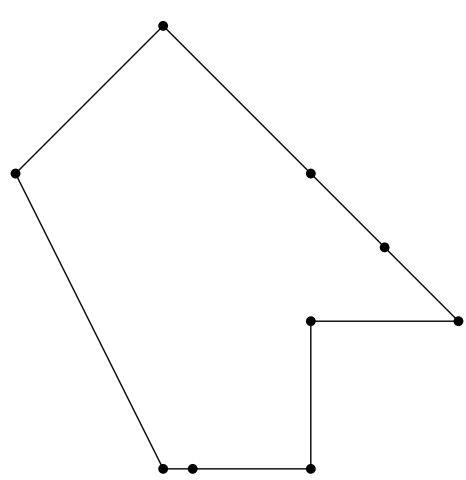

(a) The element $E$ is given

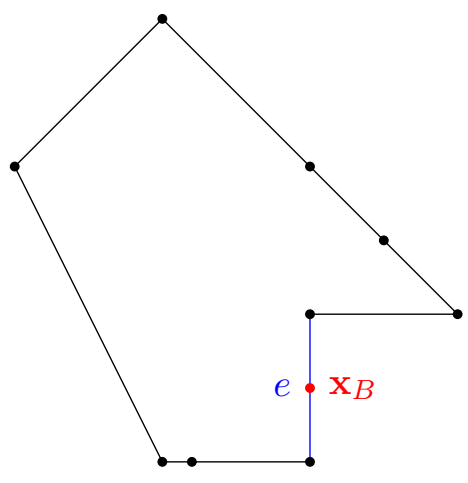

(b) The point $x_{B}$ is on the edge $e$ of the boundary $\partial E$

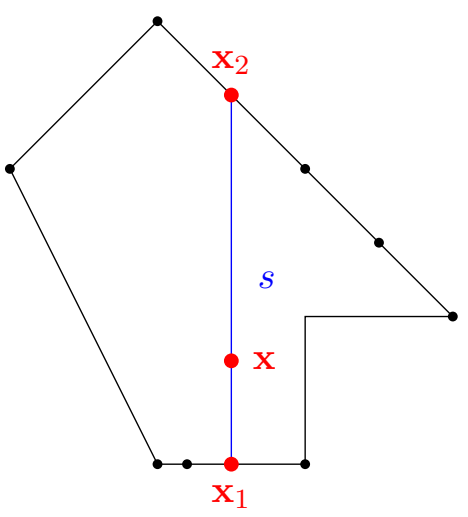

(c) $\mathbf{x}_{1}$ and $\mathbf{x}_{2}$ are two distinct intersection points between $s$ and $\partial E$ such that $\left[\mathbf{x}_{1}, \mathbf{x}_{2}\right] \subset E$

Figure 1: Some steps of the proof of Lemma 2.

that proves (40). Now let $\mathbf{x} \in \stackrel{\circ}{E}$ and let $s$ be any straight line contained in the plane of $E$ and passing through $\mathbf{x}$, let $\mathbf{x}_{1}, \mathbf{x}_{2} \in s \cap \partial E$ such that $\left[\mathbf{x}_{1}, \mathbf{x}_{2}\right] \subset E$, see Fig. $1(\mathrm{c})$, and let $d_{s}$ be the linear interpolant of $d$ on $s$. From classical Lagrange interpolation estimates and from (40) we have that

$$
\begin{aligned}
|d(\mathbf{x})| & \leq\left\|d_{s}\right\|_{L^{\infty}(s)}+\left\|d-d_{s}\right\|_{L^{\infty}(s)}=\max \left(\left|d\left(\mathbf{x}_{1}\right)\right|,\left|d\left(\mathbf{x}_{2}\right)\right|\right)+\left\|d-d_{s}\right\|_{L^{\infty}(s)} \\
& \leq\|d\|_{L^{\infty}(\partial E)}+\frac{|s|^{2}}{8}|d|_{\mathcal{C}^{2}(s)} \leq \frac{h^{2}}{8}|d|_{\mathcal{C}^{2}(E)}+\frac{h^{2}}{8}|d|_{\mathcal{C}^{2}(E)} \leq \frac{h^{2}}{4}|d|_{\mathcal{C}^{2}(E)} \leq \frac{h^{2}}{4}|d|_{\mathcal{C}^{2}(U)},
\end{aligned}
$$

where $U$ is the Fermi stripe of $\Gamma$, but $|d|_{\mathcal{C}^{2}(U)}$ depends only on the curvature of $\Gamma$, thus (41) proves (36). To prove (37), (38) and (39), we proceed as in Lemma 4.1 in [34] using estimate (36) for polygonal meshes.

The following lemma generalizes Lemma 4.2 in [34] to our polygonal setting and provides lower and upper bounds for some norms of arbitrary functions when they are unlifted from $\Gamma$ to $\Gamma_{h}$ or lifted from $\Gamma_{h}$ to $\Gamma$. In particular, it provides an equivalence between the $L^{2}\left(\Gamma_{h}\right)$ and $L^{2}(\Gamma)$ norms and between the $H^{1}\left(\Gamma_{h}\right)$ and $H^{1}(\Gamma)$ seminorms.

Lemma 3. Let $w: \Gamma_{h} \rightarrow \mathbb{R}$ with lift $w^{\ell}: \Gamma \rightarrow \mathbb{R}$. Let $\mathbf{a}: \Gamma_{h} \rightarrow \Gamma$ be the projection onto $\Gamma$ defined in (1) and, for every $E \in \mathcal{T}_{h}$, let $\tilde{E}=\mathbf{a}(E) \subset \Gamma$ be the curved triangle corresponding to $E \in \mathcal{T}_{h}$. Then

$$
\begin{aligned}
& \frac{1}{C}\|w\|_{L^{2}(E)} \leq\left\|w^{\ell}\right\|_{L^{2}(\tilde{E})} \leq C\|w\|_{L^{2}(E)} ; \\
& \frac{1}{C}\left\|\nabla_{E} w\right\|_{L^{2}(E)} \leq\left\|\nabla_{\tilde{E}} w^{\ell}\right\|_{L^{2}(\tilde{E})} \leq C\left\|\nabla_{E} w\right\|_{L^{2}(E)} ; \\
& \left\|\nabla_{E}^{2} w\right\|_{L^{2}(E)} \leq C\left\|\nabla_{\tilde{E}}^{2} w^{\ell}\right\|_{L^{2}(\tilde{E})}+C h_{E}\left\|\nabla_{\tilde{E}} w^{\ell}\right\|_{L^{2}(\tilde{E})},
\end{aligned}
$$

if the norms exist, where $C$ depends only on the surface area and the curvature of $\Gamma$.

Proof. We use the estimates of Lemma 2 and proceed exactly as in [34, Lemma 4.2].

The following result provides, in the case $k=1$, error estimates for the interpolation in $\left(V_{h}^{1}\right)^{\ell}$ and the projection on $\left(\prod_{E} \mathbb{P}_{1}(E)\right)^{\ell}$. The interpolation result extends to SVEM Lemma 4.3 in [34] for the triangular SFEM.

Theorem 6. Given a $\mathcal{C}^{2}$ surface $\Gamma$, there exists $C>0$ such that, for all $v \in H^{2}(\Gamma)$ and $w \in H^{s}(\Gamma)$, $s \in\{1,2\}$, and for all $h>0$, then 
- the interpolant $v_{I} \in V_{h}^{1}$ fulfills

$$
\left\|v-v_{I}^{\ell}\right\|_{L^{2}(\Gamma)}+h\left|v-v_{I}^{\ell}\right|_{H^{1}(\Gamma)} \leq C h^{2}\left(|v|_{H^{2}(\Gamma)}+h|v|_{H^{1}(\Gamma)}\right) ;
$$

- there exists a projection $w_{\pi} \in \prod_{E} \mathbb{P}_{1}(E)$ such that

$$
\left\|w-w_{\pi}^{\ell}\right\|_{L^{2}(\Gamma)}+h\left|w-w_{I}^{\ell}\right|_{h, 1} \leq C h^{s}\left(|w|_{H^{s}(\Gamma)}+h|w|_{H^{1}(\Gamma)}\right) .
$$

Proof. From Lemma 3, $w^{-\ell} \in H^{1}\left(\Gamma_{h}\right) \cap \prod_{E} H^{s}(E)$. Let $w_{\pi}$ be the $\prod_{E} \mathbb{P}_{1}(E)$ projection of $w^{-\ell}$ as in (34) and let $v_{I}$ be the $V_{h}^{1}$ interpolant of $v^{-\ell}$ defined piecewise by (10). From Theorems 4 and 5 , by summing piecewise contributions, we have

$$
\begin{aligned}
& \left\|w^{-\ell}-w_{\pi}\right\|_{L^{2}\left(\Gamma_{h}\right)}+h\left|w^{-\ell}-w_{\pi}\right|_{h, 1} \leq C h^{s}\left|w^{-\ell}\right|_{2, h}, \\
& \left\|v^{-\ell}-v_{I}\right\|_{L^{2}\left(\Gamma_{h}\right)}+h\left|v^{-\ell}-v_{I}\right|_{H^{1}\left(\Gamma_{h}\right)} \leq C h^{2}\left|v^{-\ell}\right|_{2, h} .
\end{aligned}
$$

From (47), (48) and Lemma 3 we have

$$
\begin{aligned}
& \left\|w-w_{\pi}^{\ell}\right\|_{L^{2}(\Gamma)}+h\left|w-w_{\pi}^{\ell}\right|_{h, 1} \leq C h^{s}\left(|w|_{H^{s}(\Gamma)}+h|w|_{H^{1}(\Gamma)}\right), \\
& \left\|v-v_{I}^{\ell}\right\|_{L^{2}(\Gamma)}+h\left|v-v_{I}^{\ell}\right|_{H^{1}(\Gamma)} \leq C h^{2}\left(|v|_{H^{2}(\Gamma)}+h|v|_{H^{1}(\Gamma)}\right),
\end{aligned}
$$

that are the desired estimates.

Remark 5. Let $\Gamma$ be a sufficiently smooth surface. If the equivalences (42)-(44) are guaranteed for arbitrary $H^{k}$ norms, the estimate (45) immediately generalizes to

$$
\left|w-w_{I}^{\ell}\right|_{H^{r}(\Gamma)} \leq C h^{k+1-r}|w|_{H^{k+1}(\Gamma)}, \quad r=0, \ldots, k,
$$

where $k$ is the order of the VEM space defined in Equation (9).

The following Lemma generalizes Lemma 4.7 in [34] to our polygonal/virtual setting and provides bounds for the geometric errors in the bilinear forms.

Lemma 4. For any $(v, w) \in H^{1}\left(\Gamma_{h}\right) \times H^{1}\left(\Gamma_{h}\right)$, the following estimates hold:

$$
\begin{array}{r}
\left|\left\langle v^{\ell}, w^{\ell}\right\rangle_{L^{2}(\Gamma)}-\langle v, w\rangle_{L^{2}\left(\Gamma_{h}\right)}\right| \leq C h^{2}\left\|v^{\ell}\right\|_{L^{2}(\Gamma)}\left\|w^{\ell}\right\|_{L^{2}(\Gamma)} \\
\left|a\left(v^{\ell}, w^{\ell}\right)-\bar{a}(v, w)\right| \leq C h^{2}\left\|\nabla_{\Gamma} v^{\ell}\right\|_{L^{2}(\Gamma)}\left\|\nabla_{\Gamma} w^{\ell}\right\|_{L^{2}(\Gamma)}
\end{array}
$$

where $C$ depends only on the geometry of $\Gamma$.

Proof. We proceed as in Lemma 4.7 of [34], but here using the generalized estimates (36)-(39) given in the previous Lemma 2.

In the first section we have recalled the Poincaré inequality $(3)$ in $H_{0}^{1}(\Gamma)$. In the following theorem we prove an analogous inequality in $H_{0}^{1}\left(\Gamma_{h}\right)$, i.e on polygonal surfaces $\Gamma_{h}$ of the type (8).

Theorem 7 (Poincaré inequality in $H_{0}^{1}\left(\Gamma_{h}\right)$ ). Let $\Gamma$ be a closed $\mathcal{C}^{2}$ orientable surface in $\mathbb{R}^{3}$. Then there exist $h_{0}>0$ and $C>0$ depending on $\Gamma$ such that, for all $0<h<h_{0}$ and $\Gamma_{h}$ as in (8),

$$
\|v\|_{L^{2}\left(\Gamma_{h}\right)} \leq C|v|_{H^{1}\left(\Gamma_{h}\right)} \quad \forall v \in H_{0}^{1}\left(\Gamma_{h}\right) .
$$

Proof. From (42) and the triangle inequality we have

$$
\|v\|_{L^{2}\left(\Gamma_{h}\right)} \leq C\left\|v^{\ell}\right\|_{L^{2}(\Gamma)} \leq C\left(\left\|v^{\ell}-\frac{1}{|\Gamma|} \int_{\Gamma} v^{\ell}\right\|_{L^{2}(\Gamma)}+\frac{1}{|\Gamma|^{\frac{1}{2}}} \int_{\Gamma} v^{\ell}\right) .
$$


Now, from (42) we have that $v^{\ell}-\frac{1}{|\Gamma|} \int_{\Gamma} v^{\ell} \in H_{0}^{1}(\Gamma)$. Then, from Poincaré's inequality (3) and (43) it follows that

$$
\left\|v^{\ell}-\frac{1}{|\Gamma|} \int_{\Gamma} v^{\ell}\right\|_{L^{2}(\Gamma)} \leq C\left|v^{\ell}\right|_{H^{1}(\Gamma)} \leq C|v|_{H^{1}\left(\Gamma_{h}\right)} .
$$

Furthermore, from (42), (51) and the fact that $v$ is zero-averaged on $\Gamma_{h}$, it follows that

$$
\frac{1}{|\Gamma|^{\frac{1}{2}}} \int_{\Gamma} v^{\ell} \leq \frac{1}{|\Gamma|^{\frac{1}{2}}}\left(\left|\int_{\Gamma_{h}} v\right|+C h^{2}\left\|v^{\ell}\right\|_{L^{2}(\Gamma)}|\Gamma|^{\frac{1}{2}}\right) \leq C h^{2}\|v\|_{L^{2}\left(\Gamma_{h}\right)} .
$$

Combining (54), (55) and (56) we have

$$
\left(1-C h^{2}\right)\|v\|_{L^{2}\left(\Gamma_{h}\right)} \leq C|v|_{H^{1}(\Gamma)} .
$$

By choosing, for instance, $h_{0}=\frac{1}{\sqrt{2 C}}$, the result follows.

Concerning the convergence rates of the above results we observe that:

- As shown in Lemma 4, in the approximation of the bilinear forms (51) and (52), the polygonal approximation of geometry yields a geometric error that is quadratic in $L^{2}$ norm and linear in $H^{1}$ norm. In fact, this Lemma is based on the geometric estimates of Lemma 2.

- The interpolation error on $\Gamma$, as shown by (45) in Lemma 6 (and its proof) arises from two sources. The first one is the interpolation error on flat polygons (cp. Lemma 4). The second one is given by the geometric estimates given in Lemma 2. Nevertheless, the order of accuracy only depends on the first of these two sources, and thus on the VEM order $k$ (cp. Remark 5).

This rate gap implies that choosing $k>1$ in (9) will not improve the convergence rate of the method, since geometric error dominates over the interpolation one. For this reason, in what follows, we restrict our study to the case $k=1$, i.e. "piecewise linear virtual elements". The same drawback occurs with the standard SFEM [34] of higher order, $k>1$; in [38] it has been shown that a finite element space of degree $k$ defined on a suitable curvilinear triangulation of degree $k$ (isoparametric elements) provides a SFEM with the same convergence rate as polynomial interpolation of degree $k$. This suggests that, to formulate a SVEM of order $k>1$, a different approximation of the surface is needed. We close this section proving an error estimate for the approximate right hand side $\left\langle f_{h}, v_{h}\right\rangle$ in the discrete formulation (33) for $k=1$.

Theorem 8. Let $f \in H_{0}^{1}(\Gamma)$. Under the regularity assumptions (A)-(B), there exists $C>0$ depending on $\Gamma$ and $\gamma$ such that

$$
\left|\left\langle f, v_{h}^{\ell}\right\rangle_{L^{2}(\Gamma)}-\left\langle f_{h}, v_{h}\right\rangle_{h}\right| \leq C h\left(|f|_{H^{1}(\Gamma)}+h|f|_{H^{2}(\Gamma)}\right)\left|v_{h}^{\ell}\right|_{H^{1}(\Gamma)} \quad \forall v_{h} \in W_{h}^{1} .
$$

Proof. Let $f_{I}$ be as in (13) and $f_{h}$ be as in (33). We split the error as

$$
\begin{aligned}
\left|\left\langle f, v_{h}^{\ell}\right\rangle_{L^{2}(\Gamma)}-\left\langle f_{h}, v_{h}\right\rangle_{h}\right| & \leq\left|\left\langle f, v_{h}^{\ell}\right\rangle_{L^{2}(\Gamma)}-\left\langle f_{I}, v_{h}\right\rangle_{L^{2}\left(\Gamma_{h}\right)}\right|+\left|\left\langle f_{I}, v_{h}\right\rangle_{L^{2}\left(\Gamma_{h}\right)}-\left\langle f_{h}, v_{h}\right\rangle_{L^{2}\left(\Gamma_{h}\right)}\right| \\
& +\left|\left\langle f_{h}, v_{h}\right\rangle_{L^{2}\left(\Gamma_{h}\right)}-\left\langle f_{h}, v_{h}\right\rangle_{h}\right| .
\end{aligned}
$$

From the Cauchy-Schwarz inequality,(51) we obtain

$$
\begin{aligned}
\left|\left\langle f, v_{h}^{\ell}\right\rangle_{L^{2}(\Gamma)}-\left\langle f_{I}, v_{h}\right\rangle_{L^{2}\left(\Gamma_{h}\right)}\right| & \leq\left|\left\langle f-f_{I}^{\ell}, v_{h}^{\ell}\right\rangle_{L^{2}(\Gamma)}\right|+\left|\left\langle f_{I}^{\ell}, v_{h}^{\ell}\right\rangle_{L^{2}(\Gamma)}-\left\langle f_{I}, v_{h}\right\rangle_{L^{2}\left(\Gamma_{h}\right)}\right| \\
& \leq\left\|f-f_{I}^{\ell}\right\|_{L^{2}(\Gamma)}\left\|v_{h}^{\ell}\right\|_{L^{2}(\Gamma)}+C h^{2}\left\|f_{I}^{\ell}\right\|_{L^{2}(\Gamma)}\left\|v_{h}^{\ell}\right\|_{L^{2}(\Gamma)}
\end{aligned}
$$

From the Cauchy-Schwarz inequality, the definition of $f_{h}$ and (51) we have

$$
\begin{aligned}
& \left|\left\langle f_{I}, v_{h}\right\rangle_{L^{2}\left(\Gamma_{h}\right)}-\left\langle f_{h}, v_{h}\right\rangle_{L^{2}\left(\Gamma_{h}\right)}\right| \leq\left|\Gamma_{h}\right|^{-\frac{1}{2}}\left|\left\langle f_{I}, 1\right\rangle_{L^{2}\left(\Gamma_{h}\right)}\right|\left\|v_{h}\right\|_{L^{2}\left(\Gamma_{h}\right)} \\
\leq & \left|\Gamma_{h}\right|^{-\frac{1}{2}}\left(\left|\left\langle f_{I}^{\ell}-f, 1\right\rangle_{L^{2}(\Gamma)}\right|+C h^{2}\left\|f_{I}^{\ell}\right\|_{L^{2}(\Gamma)}\right)\left\|v_{h}\right\|_{L^{2}\left(\Gamma_{h}\right)} \\
\leq & \left(\left\|f_{I}^{\ell}-f\right\|_{L^{2}(\Gamma)}+C h^{2}\left\|f_{I}^{\ell}\right\|_{L^{2}(\Gamma)}\right)\left\|v_{h}\right\|_{L^{2}\left(\Gamma_{h}\right)}
\end{aligned}
$$


Following [1], we know that

$$
\left|\left\langle f_{h}, v_{h}\right\rangle_{L^{2}\left(\Gamma_{h}\right)}-\left\langle f_{h}, v_{h}\right\rangle_{h}\right| \leq C h\left|f_{h}\right|_{1, h}\left|v_{h}\right|_{H^{1}\left(\Gamma_{h}\right)},
$$

but, from the definition of $f_{h}$ and from (43) it follows that

$$
\left|f_{h}\right|_{1, h}=\left|f_{I}\right|_{H^{1}\left(\Gamma_{h}\right)} \leq C\left|f_{I}^{\ell}\right|_{H^{1}(\Gamma)} .
$$

Combining (58)-(62), using (42),(43), (45), the Poincaré inequalities (3), (53) and the triangle inequality we obtain

$$
\begin{aligned}
& \left|\left\langle f, v_{h}^{\ell}\right\rangle_{L^{2}(\Gamma)}-\left\langle f_{h}, v_{h}\right\rangle_{h}\right| \leq\left(\left\|f-f_{I}^{\ell}\right\|_{L^{2}(\Gamma)}+C h\left|f_{I}^{\ell}\right|_{H^{1}(\Gamma)}+C h^{2}\left\|f_{I}^{\ell}\right\|_{L^{2}(\Gamma)}\right)\left|v_{h}^{\ell}\right|_{H^{1}(\Gamma)} \\
\leq & \left(\left(1+C h^{2}\right)\left\|f-f_{I}^{\ell}\right\|_{L^{2}(\Gamma)}+C h^{2}\|f\|_{L^{2}(\Gamma)}+C h\left|f-f_{I}^{\ell}\right|_{H^{1}(\Gamma)}+C h|f|_{H^{1}(\Gamma)}\right)\left|v_{h}^{\ell}\right|_{H^{1}(\Gamma)} \\
\leq & C\left(\left(h^{2}+h^{4}\right)|f|_{H^{2}(\Gamma)}+\left(h+h^{3}+h^{5}\right)|f|_{H^{1}(\Gamma)}\right)\left|v_{h}^{\ell}\right|_{H^{1}(\Gamma)} \leq C h\left(|f|_{H^{1}(\Gamma)}+h|f|_{H^{2}(\Gamma)}\right)\left|v_{h}^{\ell}\right|_{H^{1}(\Gamma)},
\end{aligned}
$$

that is the desired estimate.

\section{Existence, uniqueness and error analysis}

The following theorem, that is the main result of this paper, extends Theorem 3.1 in [1] for the VEM on planar domains to the Laplace-Beltrami equation on surfaces. In fact, it provides: (i) the existence and the uniqueness of the solution for both the continuous (7) and the discrete problem (33) and (ii) an abstract convergence result. As a corollary, an optimal $H^{1}(\Gamma)$ error estimate for problem (33) will be given.

Theorem 9 (Abstract convergence theorem). Let $a: H_{0}^{1}(\Gamma) \times H_{0}^{1}(\Gamma) \rightarrow \mathbb{R}$ be the bilinear form defined by

$$
a(u, v)=\int_{\Gamma} \nabla_{\Gamma} u \cdot \nabla_{\Gamma} v \quad \forall u, v \in H_{0}^{1}(\Gamma),
$$

and let $a_{h}: W_{h}^{1} \times W_{h}^{1} \rightarrow \mathbb{R}$ be any symmetric bilinear form such that

$$
a_{h}\left(u_{h}, v_{h}\right)=\sum_{E \in \mathcal{T}_{h}} a_{h, E}\left(u_{h \mid E}, v_{h \mid E}\right)
$$

where, for all $E \in \mathcal{T}_{h}, a_{h, E}$ is a symmetric bilinear form on $V_{h}^{1}(E) \times V_{h}^{1}(E)$ such that

$$
\begin{array}{ll}
\left|a_{h, E}\left(p, v_{h, E}\right)-a_{\tilde{E}}\left(p^{\ell}, v_{h, E}^{\ell}\right)\right| \leq C h^{2}\left|p^{\ell}\right|_{H^{1}(\tilde{E})}\left|v_{h, E}^{\ell}\right|_{H^{1}(\tilde{E})} & \forall v_{h, E} \in V_{h}^{1}(E) \forall p \in \mathbb{P}_{1}(E) ; \\
\alpha_{*} a_{\tilde{E}}\left(v_{h, E}^{\ell}, v_{h, E}^{\ell}\right) \leq a_{h, E}\left(v_{h, E}, v_{h, E}\right) \leq \alpha^{*} a_{\tilde{E}}\left(v_{h, E}^{\ell}, v_{h, E}^{\ell}\right) & \forall v_{h, E} \in V_{h}^{1}(E),
\end{array}
$$

where $\alpha_{*}$ and $\alpha^{*}$ are independent of $h$ and $E \in \mathcal{T}_{h}$.

Let $F \in L^{2}(\Gamma)^{\prime}$ and $F_{h} \in\left(W_{h}^{1}\right)^{\prime}$ be linear continuous functionals. Consider the problems

$$
\begin{aligned}
& \left\{\begin{array}{l}
u \in H_{0}^{1}(\Gamma) \\
a(u, v)=F(v) \quad \forall v \in H_{0}^{1}(\Gamma)
\end{array}\right. \\
& \left\{\begin{array}{l}
u_{h} \in W_{h} \\
a_{h}\left(u_{h}, v_{h}\right)=F_{h}\left(v_{h}\right) \quad \forall v_{h} \in W_{h}^{1}
\end{array}\right.
\end{aligned}
$$

Both of these problems have a unique solution and the following error estimate holds

$$
\left|u-u_{h}^{\ell}\right|_{H^{1}(\Gamma)} \leq C\left(\left|u-u_{\pi}^{\ell}\right|_{h, 1}+\left|u-u_{I}^{\ell}\right|_{H^{1}(\Gamma)}+\mathcal{F}_{h}+h\|F\|_{L^{2}(\Gamma)^{\prime}}\right),
$$

where $\mathcal{F}_{h}$ is the smallest constant such that

$$
\left|F\left(v_{h}^{\ell}\right)-F_{h}\left(v_{h}\right)\right| \leq \mathcal{F}_{h}\left|v_{h}^{\ell}\right|_{H^{1}(\Gamma)} \quad \forall v_{h} \in W_{h}^{1} .
$$


Proof. Existence and uniqueness follow from Lax-Milgram's theorem. In fact, from the Poincaré inequality (3) on $H_{0}^{1}(\Gamma)$, the bilinear form $a$ is coercive and, from the Cauchy-Schwarz inequality, it is continuous. The bilinear form $a_{h}$ is coercive since

$$
\begin{aligned}
\left|a_{h}\left(v_{h}, v_{h}\right)\right| & \underset{(63)}{=}\left|\sum_{E \in \mathcal{T}_{h}} a_{h, E}\left(v_{h \mid E}, v_{h \mid E}\right)\right| \underset{(65)}{\geq} \alpha_{*} \sum_{E \in \mathcal{T}_{h}}\left|a_{\tilde{E}}\left(v_{h \mid E}^{\ell}, v_{h \mid E}^{\ell}\right)\right|=\alpha_{*} \sum_{E \in \mathcal{T}_{h}}\left|v_{h}^{\ell}\right|_{H^{1}(\tilde{E})}^{2} \\
& \underset{(43)}{\geq} C \sum_{E \in \mathcal{T}_{h}}\left|v_{h}\right|_{H^{1}(E)}^{2}=C\left|v_{h}\right|_{H^{1}\left(\Gamma_{h}\right)}^{2} \underset{(53)}{\geq} C\left\|v_{h}\right\|_{H^{1}\left(\Gamma_{h}\right)}^{2},
\end{aligned}
$$

for all $v_{h} \in W_{h}^{1}$. Now we prove that $a_{h}$ is continuous. To this end, since $a_{h}$ is symmetric and coercive (i.e. positive definite), then it fulfills the Cauchy-Schwarz inequality. Then we have

$$
\begin{aligned}
&\left|a_{h}\left(v_{h}, w_{h}\right)\right| \underset{(63)}{\leq} \sum_{E \in \mathcal{T}_{h}}\left|a_{h, E}\left(v_{h \mid E}, w_{h \mid E}\right)\right| \leq \sum_{E \in \mathcal{T}_{h}} a_{h, E}\left(v_{h \mid E}, v_{h \mid E}\right)^{\frac{1}{2}} a_{h, E}\left(w_{h \mid E}, w_{h \mid E}\right)^{\frac{1}{2}} \\
& \underset{(65)}{\leq} \alpha^{*} \sum_{E \in \mathcal{T}_{h}} a_{\tilde{E}}\left(v_{h \mid E}^{\ell}, v_{h \mid E}^{\ell}\right)^{\frac{1}{2}} a_{\tilde{E}}\left(w_{h \mid E}^{\ell}, w_{h \mid E}^{\ell}\right)^{\frac{1}{2}}=\alpha^{*} \sum_{E \in \mathcal{T}_{h}}\left|v_{h}^{\ell}\right|_{H^{1}(\tilde{E})}\left|w_{h}^{\ell}\right|_{H^{1}(\tilde{E})} \\
& \underset{(43)}{\leq} C \sum_{E \in \mathcal{T}_{h}}\left|v_{h}\right|_{H^{1}(E)}\left|w_{h}\right|_{H^{1}(E)} \leq C\left(\sum_{E \in \mathcal{T}_{h}}\left|v_{h}\right|_{H^{1}(E)}^{2}\right)^{\frac{1}{2}}\left(\sum_{E \in \mathcal{T}_{h}}\left|w_{h}\right|_{H^{1}(E)}^{2}\right)^{\frac{1}{2}} \\
&= C\left|v_{h}\right|_{H^{1}\left(\Gamma_{h}\right)}\left|w_{h}\right|_{H^{1}\left(\Gamma_{h}\right)} \leq C\left\|v_{h}\right\|_{H^{1}\left(\Gamma_{h}\right)}|| w_{h} \|_{H^{1}\left(\Gamma_{h}\right)},
\end{aligned}
$$

for all $v_{h} \in W_{h}^{1}$. Hence, problems (66) and (67) meet the assumptions of Lax-Milgram's theorem.

If $\mathbf{a}: \Gamma_{h} \rightarrow \Gamma$ is the projection onto $\Gamma$ defined in (1), then for any $E \in \mathcal{T}_{h}$, let $\tilde{E}=\mathbf{a}(E)$ be the curved triangle corresponding to $E$. Let $u_{\pi} \in \prod_{E \in \mathcal{T}_{h}} \mathbb{P}_{1}(E)$ be the projection of $u$ defined in (46) and let $u_{I} \in W_{h}^{1}$ be the interpolant of $u$ defined in (45). From [34, Theorem 3.3], The solution of (66) fulfills $u \in H^{2}(\Gamma)$ and thus $u_{\pi}$ and $u_{I}$ are well-defined. Let $\delta_{h}=u_{h}-u_{I}$. It holds that

$$
\begin{aligned}
& \alpha_{*}\left|\delta_{h}^{\ell}\right|_{W}^{2}=\alpha_{*} a\left(\delta_{h}^{\ell}, \delta_{h}^{\ell}\right) \leq a_{h}\left(\delta_{h}, \delta_{h}\right)=a_{h}\left(u_{h}, \delta_{h}\right)-a_{h}\left(u_{I}, \delta_{h}\right) \\
& \underset{(63)}{=} F_{h}\left(\delta_{h}\right)-\sum_{E \in \mathcal{T}_{h}} a_{h, E}\left(u_{I}, \delta_{h}\right)=F_{h}\left(\delta_{h}\right)-\sum_{E \in \mathcal{T}_{h}}\left(a_{h, E}\left(u_{I}-u_{\pi}, \delta_{h}\right)+a_{h, E}\left(u_{\pi}, \delta_{h}\right)\right) \\
& \underset{(64)}{\leq} F_{h}\left(\delta_{h}\right)-\sum_{E \in \mathcal{T}_{h}}\left(a_{h, E}\left(u_{I}-u_{\pi}, \delta_{h}\right)+a_{\tilde{E}}\left(u_{\pi}^{\ell}, \delta_{h}^{\ell}\right)\right)+C h^{2} \sum_{E \in \mathcal{T}_{h}}\left|u_{\pi}^{\ell}\right|_{H^{1}(\tilde{E})}\left|\delta_{h}^{\ell}\right|_{H^{1}(\tilde{E})} \\
& =F_{h}\left(\delta_{h}\right)-\sum_{E \in \mathcal{T}_{h}}\left(a_{h, E}\left(u_{I}-u_{\pi}, \delta_{h}\right)+a_{\tilde{E}}\left(u_{\pi}^{\ell}-u, \delta_{h}^{\ell}\right)+a_{\tilde{E}}\left(u, \delta_{h}^{\ell}\right)\right)+C h^{2}\left(\left|u_{\pi}^{\ell}\right|_{h, 1}^{2}+\left|\delta_{h}^{\ell}\right|_{H^{1}(\Gamma)}^{2}\right) \\
& =F_{h}\left(\delta_{h}\right)-a\left(u, \delta_{h}^{\ell}\right)-\sum_{E \in \mathcal{T}_{h}}\left(a_{h, E}\left(u_{I}-u_{\pi}, \delta_{h}\right)+a_{\tilde{E}}\left(u_{\pi}^{\ell}-u, \delta_{h}^{\ell}\right)\right)+C h^{2}\left(\left|u_{\pi}^{\ell}\right|_{h, 1}^{2}+\left|\delta_{h}^{\ell}\right|_{H^{1}(\Gamma)}^{2}\right) \\
& =F_{h}\left(\delta_{h}\right)-F\left(\delta_{h}^{\ell}\right)-\sum_{E \in \mathcal{T}_{h}}\left(a_{h, E}\left(u_{I}-u_{\pi}, \delta_{h}\right)+a_{\tilde{E}}\left(u_{\pi}^{\ell}-u, \delta_{h}^{\ell}\right)\right)+C h^{2}\left(\left|u_{\pi}^{\ell}\right|_{h, 1}^{2}+\left|\delta_{h}^{\ell}\right|_{H^{1}(\Gamma)}^{2}\right) .
\end{aligned}
$$

From (65), (69) and the continuity of $a$ and $a_{h}$ we obtain

$$
\left(\alpha_{*}-C h^{2}\right)\left|\delta_{h}^{\ell}\right|_{H^{1}(\Gamma)}^{2} \leq \mathcal{F}_{h}\left|\delta_{h}^{\ell}\right|_{H^{1}(\Gamma)}+\left|u_{I}-u_{\pi}\right|_{h, 1}\left|\delta_{h}\right|_{H^{1}\left(\Gamma_{h}\right)}+\left.\left.\left|u_{\pi}^{\ell}-u\right|_{h, 1}\right|_{h} ^{\ell}\right|_{H^{1}(\Gamma)}+C h^{2}\left|u_{\pi}^{\ell}\right|_{h, 1}^{2}
$$

For $h$ sufficiently small, by exploiting (43), we obtain

$$
\left|\delta_{h}^{\ell}\right|_{H^{1}(\Gamma)}^{2} \leq C\left(\mathcal{F}_{h}+\left|u_{I}^{\ell}-u_{\pi}^{\ell}\right|_{h, 1}+\left|u_{\pi}^{\ell}-u\right|_{h, 1}\right)\left|\delta_{h}^{\ell}\right|_{H^{1}(\Gamma)}+C h^{2}\left|u_{\pi}^{\ell}\right|_{h, 1}^{2}
$$

By defining $A=\mathcal{F}_{h}+\left|u_{I}^{\ell}-u_{\pi}^{\ell}\right|_{h, 1}+\left|u_{\pi}^{\ell}-u\right|_{h, 1}$ and solving the second-degree-algebraic inequality (70) we have

$$
\left|\delta_{h}^{\ell}\right|_{H^{1}(\Gamma)} \leq \frac{C A}{2}+\frac{1}{2} \sqrt{C^{2} A^{2}+4 C h^{2}\left|u_{\pi}^{\ell}\right|_{h, 1}^{2}} \leq \frac{C A}{2}+\frac{1}{2}\left(C A+2 \sqrt{C} h\left|u_{\pi}^{\ell}\right|_{h, 1}\right) \leq C A+C h\left|u_{\pi}^{\ell}\right|_{h, 1} .
$$


By recalling the definition of $A$ and applying the triangle inequality, we get

$$
\left|u-u_{h}^{\ell}\right|_{H^{1}(\Gamma)} \leq C\left(\mathcal{F}_{h}+\left|u-u_{I}^{\ell}\right|_{H^{1}(\Gamma)}+\left|u-u_{\pi}^{\ell}\right|_{h, 1}\right)+C h\left|u_{\pi}^{\ell}\right|_{h, 1} .
$$

By applying the triangle inequality to the last term, we obtain

$$
\left|u-u_{h}^{\ell}\right|_{H^{1}(\Gamma)} \leq C\left(\mathcal{F}_{h}+\left|u-u_{I}^{\ell}\right|_{H^{1}(\Gamma)}+(1+h)\left|u-u_{\pi}^{\ell}\right|_{h, 1}+h|u|_{H^{1}(\Gamma)}\right) .
$$

The obvious stability estimate $|u|_{H^{1}(\Gamma)} \leq C\|F\|_{L^{2}(\Gamma)^{\prime}}$, where $C$ is the constant in the Poincaré inequality (3), together with $h \leq h_{0}$, complete the proof.

From the abstract framework given in Theorem 9 we are now ready to derive the $H^{1}(\Gamma)$ error estimate between the continuous problem (7) and the discrete one (33).

Corollary $1\left(H^{1}(\Gamma)\right.$ error estimate). Problems (7) and (33) have a unique solution. Let $u$ and $u_{h}$ be the their solutions, respectively. Under the mesh regularity assumptions $(A)-(B)$, if $f \in H_{0}^{2}(\Gamma)$, the following estimate holds:

$$
\left|u-u_{h}^{\ell}\right|_{H^{1}(\Gamma)} \leq C h\left(|u|_{H^{2}(\Gamma)}+|f|_{H^{1}(\Gamma)}\right)+C h^{2}|f|_{H^{2}(\Gamma)} .
$$

Proof. In Theorem 9, we choose

$$
\begin{array}{ll}
F(v)=\langle f, v\rangle_{L^{2}(\Gamma)} & \forall v \in H^{1}(\Gamma) ; \\
F_{h}\left(v_{h}\right)=\left\langle f_{h}, v_{h}\right\rangle_{h} & \forall v_{h} \in W_{h}^{1},
\end{array}
$$

with $a_{h}$ defined in (23), (25). Under the regularity assumptions (A)-(B),

1. Assumption (64) follows from (24) and (52);

2. Assumption (65) follows from (20), (22), (23) and (43);

3. From [34, Theorem 3.3] we have $u \in H^{2}(\Gamma)$. Then, Theorem 6 provides

$$
\left|u-u_{\pi}^{\ell}\right|_{h, 1}+\left|u-u_{I}^{\ell}\right|_{H^{1}(\Gamma)}<C h\left(|u|_{H^{2}(\Gamma)}+h|u|_{H^{1}(\Gamma)}\right) ;
$$

4. if $f \in H_{0}^{1}(\Gamma)$, The Poincaré inequality (3) provides

$$
\|F\|_{L^{2}(\Gamma)^{\prime}}=\|f\|_{L^{2}(\Gamma)} \leq C|f|_{H^{1}(\Gamma)},
$$

and (57) yields

$$
\mathcal{F}_{h} \leq C h\left(|f|_{H^{1}(\Gamma)}+h|f|_{H^{2}(\Gamma)}\right)
$$

By plugging (72)-(74), into the abstract error bound (68), we obtain

$$
\left|u-u_{h}^{\ell}\right|_{H^{1}(\Gamma)} \leq C h\left(|u|_{H^{2}(\Gamma)}+|f|_{H^{1}(\Gamma)}\right)+C h^{2}\left(|u|_{H^{1}(\Gamma)}+|f|_{H^{2}(\Gamma)}\right)
$$

By plugging the Poincaré inequality (3), the stability estimate $|u|_{H^{1}(\Gamma)} \leq C\|F\|_{L^{2}(\Gamma)^{\prime}}$ and (73) into (75), the result follows.

\section{Pasting polygonal surfaces along a straight line}

In this section we discuss a possible advantage of SVEM with respect to SFEM. Suppose that $\Gamma$ is made up of two surfaces $\Gamma_{1}$ and $\Gamma_{2}$, joining along a straight line, i.e. $\Gamma=\Gamma_{1} \cup \Gamma_{2}$ and $\Gamma_{1} \cap \Gamma_{2}=\ell$ is a straight line. Furthermore, suppose we are given two corresponding polygonal surfaces $\Gamma_{1, h}, \Gamma_{2, h}$, and that these polygonal surfaces fit $\ell$ exactly, i.e. $\Gamma_{1, h} \cap \Gamma_{2, h}=\ell$. We want to construct a polygonal surface $\Gamma_{h}$ by pasting $\Gamma_{h, 1}$ and $\Gamma_{h, 2}$. Such a process is depicted in Fig. 2 and leads, in general, to a nonconforming overall triangulation. For this reason, the possibility of handling hanging nodes is crucial in this pasting process. It is well-known that the triangular FEMs, including SFEM, are not applicable to nonconforming meshes. For this reason, pasting algorithms for standard FEMs typically need additional deforming and node matching steps, see for instance $[12,13]$. 


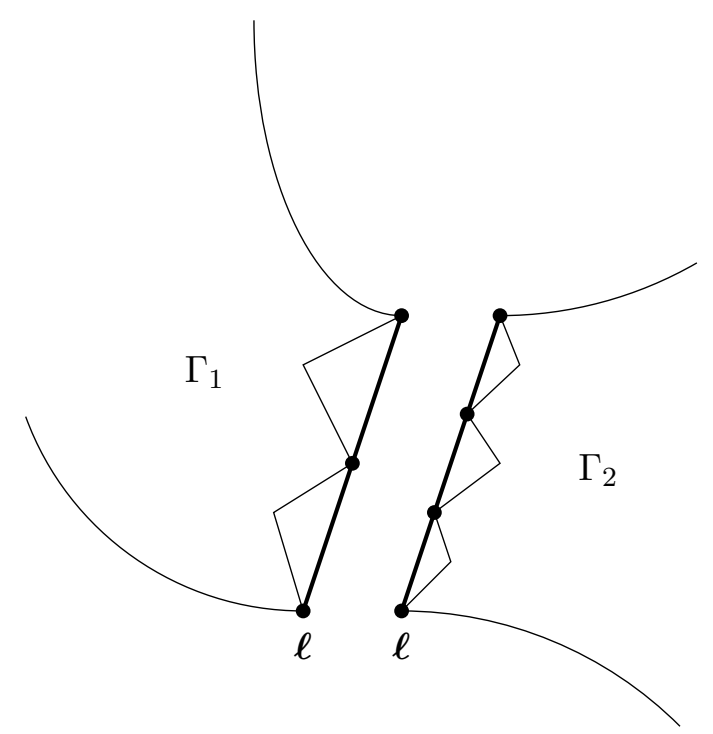

(a) Step 1

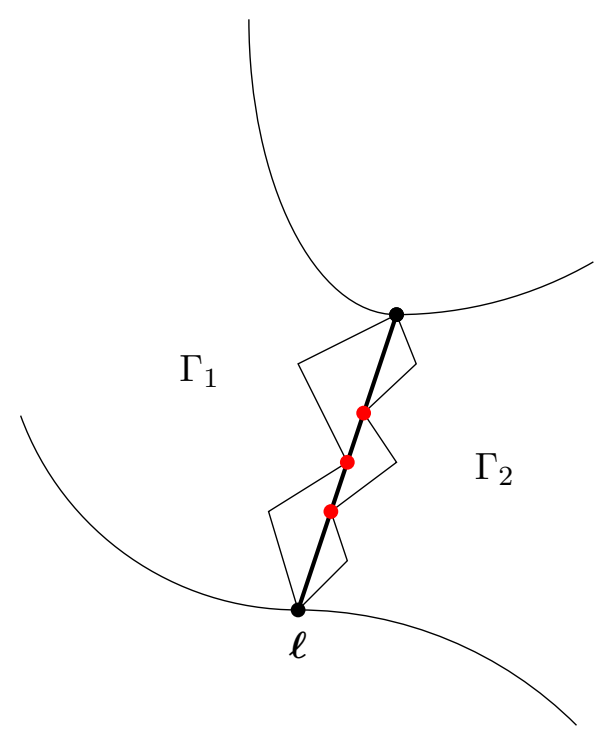

(b) Step 2

Figure 2: Pasting algorithm. Step 1: two surfaces $\Gamma_{1}$ and $\Gamma_{2}$ are given together with their approximations $\Gamma_{1, h}$ and $\Gamma_{2, h}$. The triangles having an edge on $\ell$ are depicted and their nodes on $\ell$ are black-marked. Step 2: by pasting the triangulated surfaces, a nonconforming polygonation of $\Gamma=\Gamma_{1} \cup \Gamma_{2}$ is formed, due to the presence of hanging nodes on $\ell$, that are red-marked.

\section{Implementation}

In this section we will discuss how to implement the SVEM for $k=1$ using only information on the mesh and the nodal values of the load term $f$. We will not consider the case $k \geq 2$ because, as discussed in Section 4, increasing $k$ does not improve the convergence rate of the method.

We observe that, from (12) and (32), problem (33) is equivalent to

$$
\left\{\begin{array}{l}
u_{h} \in V_{h}^{k} \\
a_{h}\left(u_{h}, \phi_{h}\right)=\left\langle f_{h}, \phi_{h}\right\rangle_{h} \quad \forall \phi_{h} \in V_{h}^{k} \\
\left\langle u_{h}, 1\right\rangle_{L^{2}\left(\Gamma_{h}\right)}=0 .
\end{array}\right.
$$

Notice that, since $k=1$, the overall number of degrees of freedom is equal to the number of nodal points. Now, for every $i=1, \ldots, N$, let $\phi_{i} \in V_{h}^{1}$ be the $i$-th basis function defined by $\operatorname{dof}_{j}\left(\phi_{i}\right)=\delta_{i j}$, for all $j=1, \ldots, N$. We express the numerical solution of (76) in the basis $\left\{\phi_{i}\right\}_{i=1}^{N}$ as

$$
u_{h}(\mathbf{x})=\sum_{j=1}^{N} \xi_{j} \phi_{j}(\mathbf{x}), \quad \forall \mathbf{x} \in \Gamma_{h},
$$

with $\xi_{j} \in \mathbb{R}$ for all $j=1, \ldots, N$. Problem (33) is then equivalent to

$$
\begin{aligned}
& \sum_{j=1}^{N} a_{h}\left(\phi_{i}, \phi_{j}\right) \xi_{j}=\left\langle f_{h}, \phi_{i}\right\rangle_{h}, \quad \forall i=1, \ldots, N, \\
& \sum_{j=1}^{N}\left\langle 1, \phi_{j}\right\rangle_{L_{h}^{2}} \xi_{j}=0 .
\end{aligned}
$$

Problem (77)-(78) is a rectangular $(N+1) \times N$ linear system that has, from Corollary 1 , a unique solution. We want to rephrase this problem as a square $N \times N$ linear system. To this end, notice that 
the function $\bar{\phi}:=\sum_{i=1}^{N} \phi_{i}$ fulfills $\operatorname{dof}_{j}(\bar{\phi})=1$ for all $j=1, \ldots, N$ and thus, from (9), we have

$$
\sum_{i=1}^{N} \phi_{i}(\mathbf{x})=1, \quad \forall \mathbf{x} \in \Gamma_{h}
$$

We show that the sum of all equations in (77) vanishes. In fact, for the left hand side of (77), using (24) and (79), we have that

$$
\sum_{i=1}^{N} \sum_{j=1}^{N} a_{h}\left(\phi_{i}, \phi_{j}\right) \xi_{j}=\sum_{j=1}^{N} a_{h}\left(\sum_{i=1}^{N} \phi_{i}, \phi_{j}\right) \xi_{j}=\sum_{j=1}^{N} a_{h}\left(1, \phi_{j}\right) \xi_{j}=\sum_{j=1}^{N} \bar{a}\left(1, \phi_{j}\right) \xi_{j}=0,
$$

while for the right hand side of (77), from (32) and (79) we have

$$
\sum_{i=1}^{N}\left\langle f_{h}, \phi_{i}\right\rangle_{h}=\left\langle f_{h}, 1\right\rangle_{h}=0
$$

We conclude that the sum of equations (77) vanishes. This implies that we can remove, for instance, the $N$-th equation in (77). System (77)-(78) is then equivalent to the $N \times N$ system

$$
\left\{\begin{array}{l}
\sum_{j=1}^{N} a_{h}\left(\phi_{i}, \phi_{j}\right) \xi_{j}=\left\langle f_{h}, \phi_{i}\right\rangle_{h}, \quad \forall i=1, \ldots, N-1, \\
\sum_{j=1}^{N}\left\langle 1, \phi_{j}\right\rangle_{L_{h}^{2}} \xi_{j}=0 .
\end{array}\right.
$$

Consider the stiffness matrix $A$, the mass matrix $M$, and the load term $\mathbf{b}$ defined by

$$
\begin{aligned}
& A=\left(a_{i j}\right):=a_{h}\left(\phi_{i}, \phi_{j}\right), \quad \forall i, j=1, \ldots, N, \\
& M=\left(m_{i j}\right):=\left\langle\phi_{i}, \phi_{j}\right\rangle_{L_{h}^{2}}, \quad \forall i, j=1, \ldots, N, \\
& \mathbf{b}=\left(b_{i}\right):=\left\langle f_{h}, \phi_{i}\right\rangle_{h}, \quad \forall i=1, \ldots, N,
\end{aligned}
$$

and, for every $E \in \mathcal{T}_{h}$, consider the local mass matrix $M_{E}$ defined by

$$
M_{E}=\left(m_{i j}^{E}\right):=\left\langle\phi_{i}, \phi_{j}\right\rangle_{L_{h}^{2}, E}, \quad \forall i, j: \mathbf{x}_{i}, \mathbf{x}_{j} \in \operatorname{nodes}(E) .
$$

The matrices $A$ and $M_{E}$ and $M$ can be computed as detailed in [8]. To compute the load vector $\mathbf{b}$ we observe that, from (30) and the definition of the basis functions, it holds that

$$
b_{i}=\sum_{E: \mathbf{x}_{i} \in \operatorname{nodes}(E)} \frac{1}{n_{E}} \int_{E} f_{h}, \quad \forall i=1, \ldots, N .
$$

We are left to compute the integrals in (80) as follows. The nodal values of the load term $f_{h}$ are computed by

$$
f_{h}\left(\mathbf{x}_{k}\right)=f_{I}\left(\mathbf{x}_{k}\right)-\frac{\left\langle f_{I}, 1\right\rangle_{L_{h}^{2}}}{\left|\Gamma_{h}\right|}=f\left(\mathbf{x}_{k}\right)-\frac{\sum_{i=1}^{N}\left\langle\phi_{i}, 1\right\rangle_{L_{h}^{2}} f\left(\mathbf{x}_{i}\right)}{\langle 1,1\rangle_{L_{h}^{2}}}=f\left(\mathbf{x}_{k}\right)-\frac{\sum_{i=1}^{N}\left(\sum_{j=1}^{N} m_{i j}\right) f\left(\mathbf{x}_{i}\right)}{\sum_{i=1}^{N} \sum_{j=1}^{N} m_{i j}},
$$

for all $k=1, \ldots, N$. For every $E$, the integral of $f_{h}$ on $E$ is given by

$$
\int_{E} f_{h} \underset{(27)}{=}\left\langle f_{h}, 1\right\rangle_{L_{h}^{2}, E}=\sum_{i: \mathbf{x}_{i} \in \operatorname{nodes}(E)}\left\langle\phi_{I}, 1\right\rangle_{L_{h}^{2}, E} f_{h}\left(\mathbf{x}_{i}\right)=\sum_{i: \mathbf{x}_{i} \in \operatorname{nodes}(E)}\left(\sum_{j: \mathbf{x}_{j} \in \operatorname{nodes}(E)} m_{i j}^{E}\right) f_{h}\left(\mathbf{x}_{i}\right) .
$$


In conclusion, the discretisation of the Laplace-Beltrami equation (5) by SVEM is given by the following sparse, square, full-rank linear algebraic system

$$
\left\{\begin{array}{l}
\sum_{j=1}^{N} a_{i j} \xi_{j}=b_{i}, \quad \forall i=1, \ldots, N-1, \\
\sum_{j=1}^{N}\left(\sum_{i=1}^{N} m_{i j}\right) \xi_{j}=0
\end{array}\right.
$$

For instance, the matrix form of system (81) in MATLAB language is given by

$$
\mathrm{xi}=[\mathrm{A}(1: \mathrm{N}-1,:) ; \operatorname{ones}(1, \mathrm{~N}) * \mathrm{M}] \backslash[\mathrm{b}(1: \mathrm{N}-1) ; 0] \text {. }
$$

\section{Numerical examples}

In this section we will validate the theoretical findings through numerical experiments.

In Experiment 1, a Laplace-Beltrami problem on the unit sphere, approximated with a polygonal mesh, is used as a benchmark to test the convergence rate in (71). The experiment also shows the robustness of the method with respect to "badly shaped" meshes, i.e with polygons of very different size and very tight, thus confirming the generality of assumptions (A)-(B). In Experiment 2, we show an example of mesh pasting, as discussed in Section 6.

\subsection{Experiment 1}

In this experiment we solve the Laplace-Beltrami equation

$$
\left\{\begin{array}{c}
-\Delta_{\Gamma} u(x, y, z)=6 x y, \quad(x, y, z) \in \Gamma, \\
\int_{\Gamma} u(x, y, z) \mathrm{d} \sigma=0
\end{array}\right.
$$

on the unit sphere $\Gamma:=\left\{(x, y, z) \in \mathbb{R}^{3} \mid x^{2}+y^{2}+z^{2}=1\right\}$, whose exact solution is given by

$$
u(x, y, z)=x y, \quad(x, y, z) \in \Gamma .
$$

We solve the problem on a sequence of seven polygonal meshes, with decreasing meshsize $h$, made up with triangles and hexagons whose vertices lie on $\Gamma$ and we test the convergence rate as follows. Let $u_{I}$ be the interpolant, defined in (45), of the exact solution $u$ and let $\delta_{h}:=u_{I}-u_{h}$. We consider the following approximations of the $L^{2}, L^{\infty}$ and $H^{1}$ errors, respectively:

$$
\begin{aligned}
& \mathrm{E}_{L^{2}, h}:=\left\langle\delta_{h}, \delta_{h}\right\rangle_{L_{h}^{2}} ; \\
& \mathrm{E}_{L^{\infty}, h}:=\max _{P \in \operatorname{nodes}\left(\Gamma_{h}\right)}\left(\delta_{h}\right) ; \\
& \mathrm{E}_{H^{1}, h}:=a_{h}\left(\delta_{h}, \delta_{h}\right),
\end{aligned}
$$

where the forms $a_{h}(\cdot, \cdot)$ and $\langle\cdot, \cdot\rangle_{L_{h}^{2}}$ are defined in (25) and (28), respectively. These approximations are $\mathcal{O}\left(h^{2}\right)$-accurate, see for instance [4]. The need of defining these approximate norms and seminorms arise from the presence of the virtual basis functions that are not known in closed form. These norms are reminiscent of the approximate $L^{2}$ norm used for instance in [4, Equation 46], but also account for the fact that, in our case, the exact and the numerical solutions are defined on different surfaces. The convergence rate in the norms and seminorms defined in (83)-(85) is estimated by computing these errors as functions of $h$.

The coarsest of the polygonal meshes under consideration (meshsize $h=0.6209$ ) is shown in Figure $3(\mathrm{a})$. The numerical solution obtained on the finest mesh (meshsize $h=0.0798$ ) is shown in Figure 3(b). The convergence results are shown in Fig. 4. The convergence is linear in $H^{1}$ norm and, even if the method is not designed for optimal $L^{2}$ and $L^{\infty}$ convergence, it appears to be quadratic in $L^{2}$ norm and almost quadratic in $L^{\infty}$ norm. We remark that the considered meshes, like the one in Fig. $3(\mathrm{a})$, have polygons of very different size and shape, this means that the regularity assumptions (A) and (B) are quite weak and the method is thus robust with respect to badly shaped meshes. 


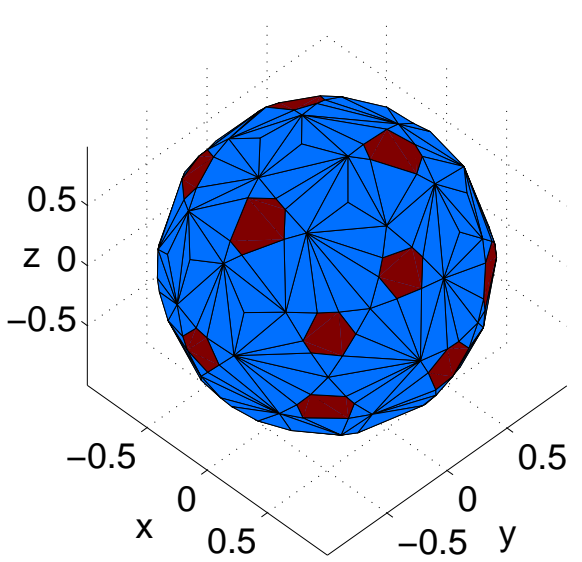

(a) Polygonal approximation $\Gamma_{h}$ of the unit sphere $\Gamma$, made up of triangles and hexagons, with meshsize $h=$ 0.4013 .

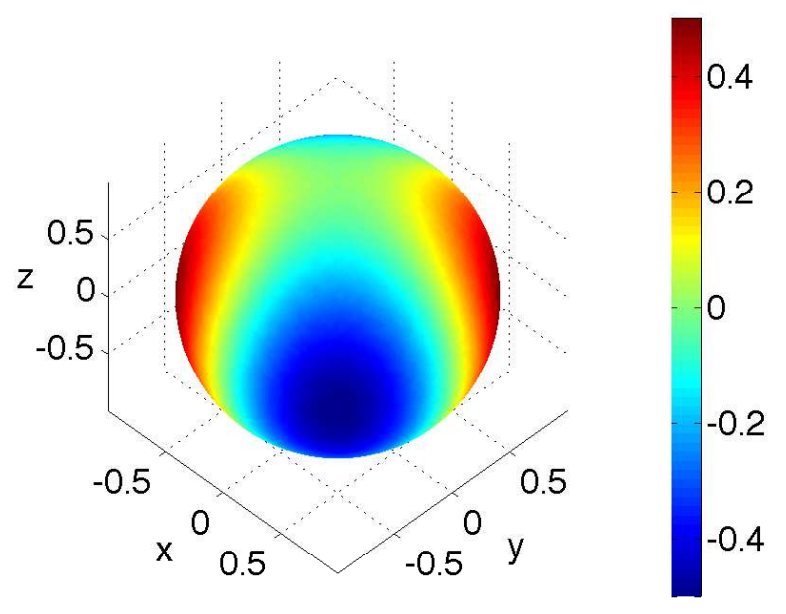

(b) Numerical solution obtained on $\Gamma_{h}$, for $h=0.0798$.

Figure 3: (a) The coarsest mesh used for the convergence study; (b) the numerical solution of problem (82) on the finest mesh.

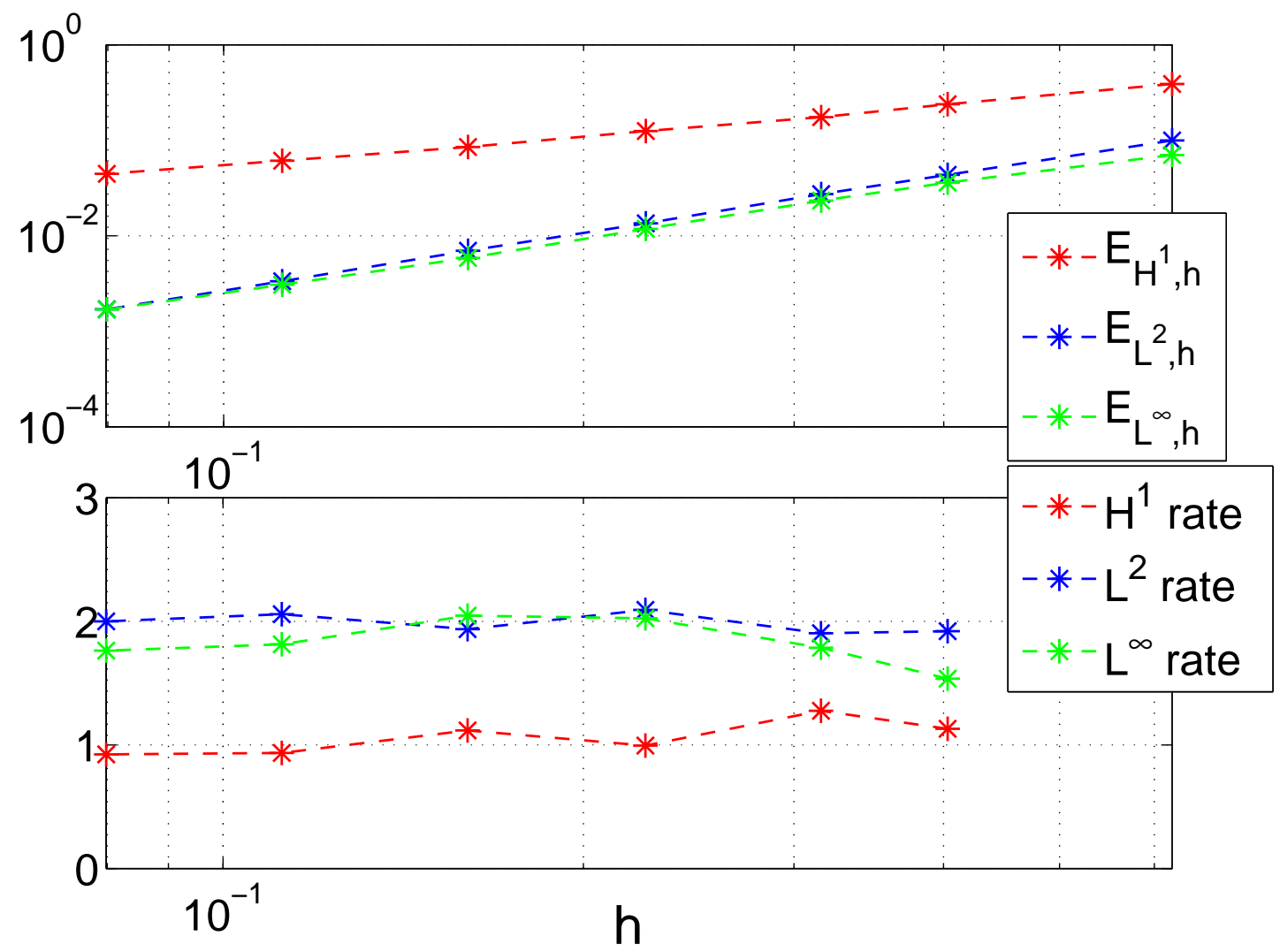

Figure 4: Convergence results for problem (82). 


\subsection{Experiment 2}

In this experiment we solve the Laplace-Beltrami equation and we address the problem of pasting two surfaces. We start by considering the cylinder

$$
\Gamma:=\left\{(x, y, z) \in \mathbb{R}^{3} \mid x^{2}+y^{2}=1 \wedge 0 \leq z \leq 2\right\}
$$

and we split it into two parts

$$
\begin{aligned}
& \Gamma_{1}:=\Gamma \cap\{y \geq 0\} \\
& \Gamma_{2}:=\Gamma \cap\{y \leq 0\} .
\end{aligned}
$$

We consider the Laplace-Beltrami equation

$$
\left\{\begin{aligned}
-\Delta_{\Gamma} u & =\left(y-x^{2}\right) e^{y} \quad \text { on } \Gamma, \\
u & =\bar{u} \text { on } \partial \Gamma,
\end{aligned}\right.
$$

where $\bar{u}$ is the exact solution, given by

$$
\bar{u}(x, y, z)=e^{y}+z, \quad(x, y, z) \in \Gamma .
$$

Notice that the considered surface has a non-empty boundary and the boundary conditions are of homogeneous Dirichlet type, see Remark 1. We consider a family of meshes defined as follows. Let $N \in \mathbb{N}$. The half cylinder $\Gamma_{1}$ is approximated with $8 N^{2}$ equal rectangular elements constructed on the following $(4 N+1)(2 N+1)$ gridpoints

$$
P_{i j}=\left(\cos \frac{i}{4 N} \pi, \sin \frac{i}{4 N} \pi, \frac{j}{N}\right), \quad i=0, \ldots 4 N, \quad j=0, \ldots, 2 N,
$$

while the half cylinder $\Gamma_{2}$ is approximated with $2 N^{2}$ equal rectangular elements constructed on the following $(2 N+1)(N+1)$ gridpoints:

$$
P_{i j}=\left(\cos \left(\frac{i}{2 N}+1\right) \pi, \sin \left(\frac{i}{2 N}+1\right) \pi, \frac{2 j}{N}\right), \quad i=0, \ldots 2 N, \quad j=0, \ldots, N .
$$

By pasting these meshes we end up with a nonconforming mesh $\Gamma_{h}$ made up of $10 N^{2}$ elements, of which $2 N(5 N-1)$ rectangles and $2 N$ degenerate pentagons with one hanging node each. For $N=10$, this nonconforming mesh is shown in Fig. 5(a), in which the rectangles are green and the pentagons are orange while the corresponding numerical solution is shown in Fig. 5(b).

To test the convergence, we consider a sequence of six meshes of the type described above, by increasing $N=5 i, i=1, \ldots, 6$. Notice that the relation between $N$ and the meshsize $h$ is

$$
h=2 \sin \left(\frac{\pi}{8 N}\right),
$$

and thus $h=\mathcal{O}\left(\frac{1}{N}\right)$. For all $i=1, \ldots, 6$, the errors in the norms and seminorms (83)-(85) are shown in Fig. 6 as functions of $h$. The experimental convergence rate is quadratic in the approximate $L^{2}$ and $L^{\infty}$ norms and superlinear in the approximate $H^{1}$ seminorm.

\section{Conclusions}

In this study, we have considered a Surface Virtual Element Method (SVEM) for the numerical approximation of the Laplace-Beltrami equation on smooth surfaces, by generalising the VEM on planar domains [1] and the SFEM [34]. By extending the results in [1] and [34] we have shown, under minimal regularity assumptions for the mesh, optimal asymptotic error estimates (i) for the interpolation in the SVEM function space, (ii) for the approximation of the surface and (iii) for the projection onto 


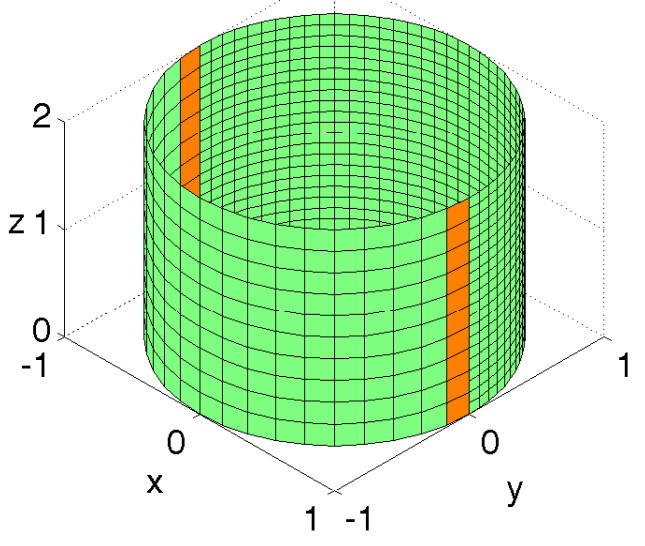

(a) Nonconforming polygonal approximation $\Gamma_{h}$ of $\Gamma$, with meshsize $h=0.2542$, made up of rectangles and pentagons.

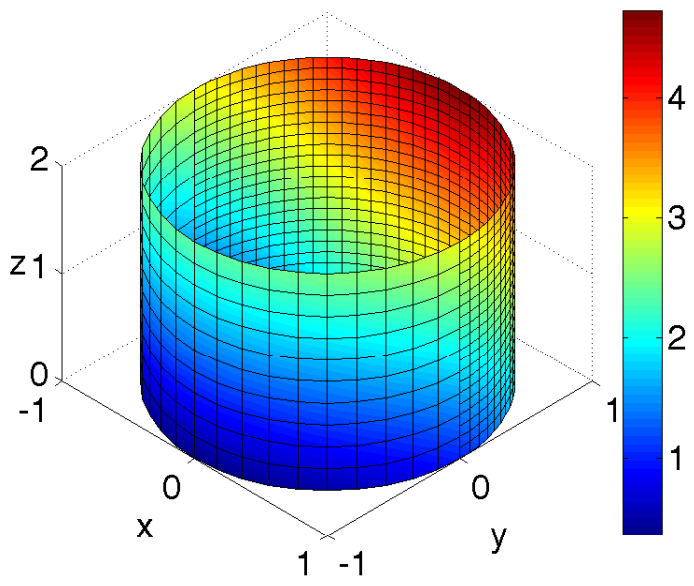

(b) Numerical solution obtained on the mesh $\Gamma_{h}$.

Figure 5: Polygonal approximation of the cylinder (86) and corresponding numerical solution of Problem (87).

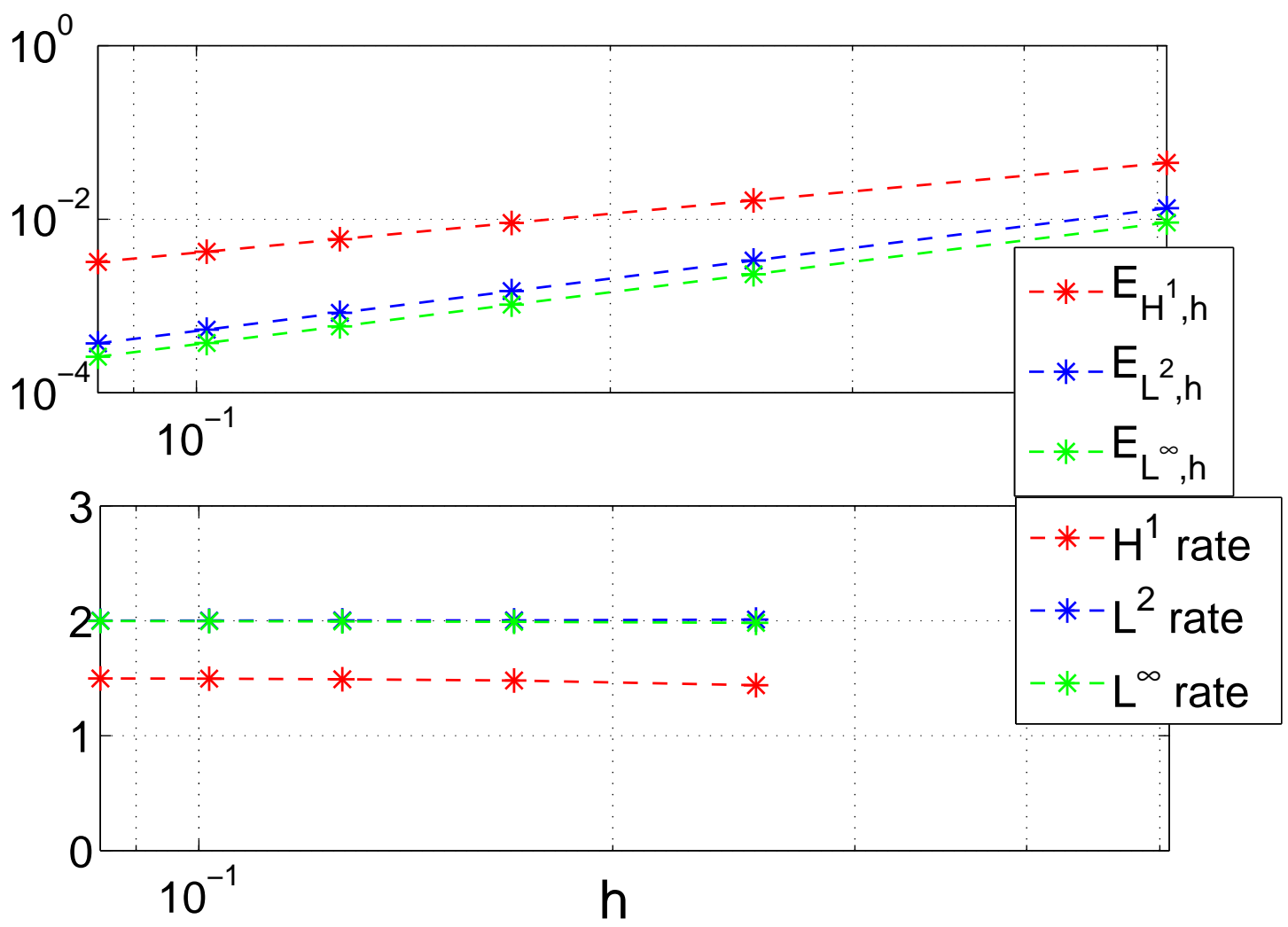

Figure 6: Convergence results for problem (87). 
the polygonal surface. In particular, the geometric error arising from the approximation of the surface is quadratic in the meshsize and is thus asymptotically dominant with respect to the interpolation error for higher polynomial orders $k>1$. Improving the geometric error is necessary to increase the convergence rate of the overall method. To this end, following [38], curved polygonal elements could be used. This will be subject of future investigations. We have also shown the existence and uniqueness of the numerical solution and, for $k=1$, its first order $H^{1}$ convergence. To highlight and advantage of the SVEM technique, we have shown that the process of pasting two meshes along a straight line leads to a nonconforming mesh that can be easily handled by the SVEM. We have presented two numerical examples for the Laplace-Beltrami equation to (i) test the convergence rate of the SVEM method for $k=1$ on the unit sphere and (ii) to show its application to mesh pasting on a cylindrical surface. Since the Laplace-Beltrami equation is endowed with the zero average condition, we have shown, for $k=1$, how to include this condition in the implementation, obtaining a sparse, square, full-rank linear algebraic system, using only information on the mesh and the nodal values of the load term. The Laplace-Beltrami equation has been considered because it is the simplest PDE defined on a surface. Given the satisfactory behavior of the SVEM on this test problem, the authors believe that it is worth extending the methodology to more complicated surface PDEs. This will be done in future studies.

\section{References}

[1] L Beirão da Veiga, F Brezzi, A Cangiani, G Manzini, L D Marini, and A Russo. Basic principles of virtual element methods. Mathematical Models and Methods in Applied Sciences, 23(01):199-214, 2013.

[2] L Beirão Da Veiga, F Brezzi, and L D Marini. Virtual elements for linear elasticity problems. SIAM Journal on Numerical Analysis, 51(2):794-812, 2013.

[3] D Mora, G Rivera, and R Rodríguez. A virtual element method for the Steklov eigenvalue problem. Mathematical Models and Methods in Applied Sciences, 25(08):1421-1445, 2015.

[4] G Vacca and L Beirão da Veiga. Virtual element methods for parabolic problems on polygonal meshes. Numerical Methods for Partial Differential Equations, 31(6):2110-2134, 2015.

[5] G Vacca. Virtual element methods for hyperbolic problems on polygonal meshes. Computers $\&$ Mathematics with Applications, 2016.

[6] P F Antonietti, L Beirão Da Veiga, S Scacchi, and M Verani. A $C^{1}$ virtual element method for the Cahn-Hilliard equation with polygonal meshes. SIAM Journal on Numerical Analysis, 54(1):34-56, 2016.

[7] M F Benedetto, S Berrone, and S Scialò. A globally conforming method for solving flow in discrete fracture networks using the virtual element method. Finite Elements in Analysis and Design, 109:23-36, 2016.

[8] L Beirão da Veiga, F Brezzi, L D Marini, and A Russo. The hitchhiker's guide to the virtual element method. Mathematical Models and Methods in Applied Sciences, 24(08):1541-1573, 2014.

[9] B Ayuso de Dios, K Lipnikov, and G Manzini. The nonconforming virtual element method. ESAIM: Mathematical Modelling and Numerical Analysis, 50(3):879-904, 2016.

[10] N Benkemoun, A Ibrahimbegovic, and J-B Colliat. Anisotropic constitutive model of plasticity capable of accounting for details of meso-structure of two-phase composite material. Computers \&5 Structures, 90:153-162, 2012.

[11] J Chen. A memory efficient discontinuous Galerkin finite-element time-domain scheme for simulations of finite periodic structures. Microwave and Optical Technology Letters, 56(8):1929-1933, 2014. 
[12] T Kanai, H Suzuki, J Mitani, and F Kimura. Interactive mesh fusion based on local 3d metamorphosis. In Graphics Interface, volume 99, pages 148-156, 1999.

[13] A Sharf, M Blumenkrants, A Shamir, and D Cohen-Or. Snappaste: an interactive technique for easy mesh composition. The Visual Computer, 22(9-11):835-844, 2006.

[14] A Cangiani, E H Georgoulis, and S Metcalfe. Adaptive discontinuous Galerkin methods for nonstationary convection-diffusion problems. IMA Journal of Numerical Analysis, 34(4):15781597, 2014.

[15] KY Dai, GR Liu, and TT Nguyen. An n-sided polygonal smoothed finite element method (nSFEM) for solid mechanics. Finite Elements in Analysis and Design, 43(11):847-860, 2007.

[16] L Beirão da Veiga and G Manzini. A virtual element method with arbitrary regularity. IMA Journal of Numerical Analysis, page drt018, 2013.

[17] F Brezzi and L D Marini. Virtual element methods for plate bending problems. Computer Methods in Applied Mechanics and Engineering, 253:455-462, 2013.

[18] N Flyer and G B Wright. Transport schemes on a sphere using radial basis functions. Journal of Computational Physics, 226(1):1059-1084, 2007.

[19] N Flyer and Grady B Wright. A radial basis function method for the shallow water equations on a sphere. In Proceedings of the Royal Society of London A: Mathematical, Physical and Engineering Sciences, pages rspa-2009. The Royal Society, 2009.

[20] P Tang, Feng Q, H Zhang, and Y Yang. Phase separation patterns for diblock copolymers on spherical surfaces: A finite volume method. Physical Review E, 72(1):016710, 2005.

[21] M Bertalmı, L T Cheng, S Osher, and G Sapiro. Variational problems and partial differential equations on implicit surfaces. Journal of Computational Physics, 174(2):759-780, 2001.

[22] M Bergdorf, I F Sbalzarini, and P Koumoutsakos. A lagrangian particle method for reactiondiffusion systems on deforming surfaces. Journal of Mathematical Biology, 61(5):649-663, 2010.

[23] R Barreira, Charles M Elliott, and A Madzvamuse. The surface finite element method for pattern formation on evolving biological surfaces. Journal of Mathematical Biology, 63(6):1095-1119, 2011.

[24] E J Fuselier and G B Wright. A high-order kernel method for diffusion and reaction-diffusion equations on surfaces. Journal of Scientific Computing, 56(3):535-565, 2013.

[25] M Frittelli, A Madzvamuse, I Sgura, and C Venkataraman. Lumped finite element method for reaction-diffusion systems on compact surfaces. arXiv preprint arXiv:1609.02741, 2016.

[26] M AJ Chaplain, M Ganesh, and I G Graham. Spatio-temporal pattern formation on spherical surfaces: numerical simulation and application to solid tumour growth. Journal of Mathematical Biology, 42(5):387-423, 2001.

[27] C M Elliott and B Stinner. Modeling and computation of two phase geometric biomembranes using surface finite elements. Journal of Computational Physics, 229(18):6585-6612, 2010.

[28] C M Elliott, B Stinner, and C Venkataraman. Modelling cell motility and chemotaxis with evolving surface finite elements. Journal of The Royal Society Interface, page rsif20120276, 2012.

[29] Q Du and L Ju. Approximations of a Ginzburg-Landau model for superconducting hollow spheres based on spherical centroidal Voronoi tessellations. Mathematics of Computation, 74(251):12571280, 2005. 
[30] C Eilks and C M Elliott. Numerical simulation of dealloying by surface dissolution via the evolving surface finite element method. Journal of Computational Physics, 227(23):9727-9741, 2008.

[31] G Xu, Q Pan, and C L Bajaj. Discrete surface modelling using partial differential equations. Computer Aided Geometric Design, 23(2):125-145, 2006.

[32] M E Taylor. Partial differential equations III: Nonlinear Equations, 2nd Ed., Series: Applied Mathematical Sciences, Vol. 11\%. Springer, 2011.

[33] C B Macdonald and S J Ruuth. The implicit closest point method for the numerical solution of partial differential equations on surfaces. SIAM Journal on Scientific Computing, 31(6):43304350, 2009.

[34] G Dziuk and C M Elliott. Finite element methods for surface PDEs. Acta Numerica, 22:289-396, 2013.

[35] N Tuncer, A Madzvamuse, and AJ Meir. Projected finite elements for reaction-diffusion systems on stationary closed surfaces. Applied Numerical Mathematics, 96:45-71, 2015.

[36] G Dziuk. Finite elements for the Beltrami operator on arbitrary surfaces. Partial Differential Equations and Calculus of Variations, pages 142-155, 1988.

[37] M E Taylor. Partial differential equations I: Basic Theory, 2nd Ed., Series: Applied Mathematical Sciences, Vol. 115. Springer, 2011.

[38] A Demlow. Higher-order finite element methods and pointwise error estimates for elliptic problems on surfaces. SIAM Journal on Numerical Analysis, 47(2):805-827, 2009. 